\title{
Observed Faraday Effects in Damped Ly $\alpha$ Absorbers and Lyman Limit Systems: The Magnetized Environment of Galactic Building Blocks at Redshift $=2$
}

\author{
J. S. Farnes ${ }^{1}$, L. Rudnick ${ }^{2}$, B. M. Gaensler ${ }^{3}$, M. Haverkorn ${ }^{1}$, S. P. O’Sullivan ${ }^{4}$, and S. J. Curran ${ }^{5}$ \\ ${ }^{1}$ Department of Astrophysics/IMAPP, Radboud University, P.O. Box 9010, NL-6500 GL Nijmegen, The Netherlands; j.farnes@astro.ru.nl \\ ${ }^{2}$ Minnesota Institute for Astrophysics, School of Physics and Astronomy, University of Minnesota, 116 Church Street SE, Minneapolis, MN 55455, USA \\ ${ }^{3}$ Dunlap Institute for Astronomy and Astrophysics, University of Toronto, ON M5S 3H4, Canada \\ ${ }^{4}$ Instituto de Astronomía, Universidad Nacional Autónoma de México (UNAM), A.P. 70-264, 04510 México, D.F., Mexico \\ ${ }^{5}$ School of Chemical and Physical Sciences, Victoria University of Wellington, P.O. Box 600, Wellington 6140, New Zealand \\ Received 2016 September 6; revised 2017 April 19; accepted 2017 April 28; published 2017 May 25
}

\begin{abstract}
Protogalactic environments are typically identified using quasar absorption lines and can manifest as Damped Lyman-alpha Absorbers (DLAs) and Lyman Limit Systems (LLSs). We use radio observations of Faraday effects to test whether these galactic building blocks host a magnetized medium, by combining DLA and LLS detections with $1.4 \mathrm{GHz}$ polarization data from the NRAO VLA Sky Survey (NVSS). We obtain a control, a DLA, and an LLS sample consisting of 114, 19, and 27 lines of sight, respectively. Using a Bayesian framework and weakly informative priors, we are unable to detect either coherent or random magnetic fields in DLAs: the regular coherent fields must be $\leqslant 2.8 \mu \mathrm{G}$, and the lack of depolarization suggests the weakly magnetized gas in DLAs is nonturbulent and quiescent. However, we find a mild suggestive indication that LLSs have coherent magnetic fields, with a $71.5 \%$ probability that LLSs have higher $|\mathrm{RM}|$ than a control, although this is sensitive to the redshift distribution. We also find a strong indication that LLSs host random magnetic fields, with a $95.5 \%$ probability that LLS lines of sight have lower polarized fractions than a control. The regular coherent fields within the LLSs must be $\leqslant 2.4 \mu \mathrm{G}$, and the magnetized gas must be highly turbulent with a typical turbulent length scale on the order of $\approx 5-20 \mathrm{pc}$. Our results are consistent with the standard dynamo paradigm, whereby magnetism in protogalaxies increases in coherence over cosmic time, and with a hierarchical galaxy formation scenario, with the DLAs and LLSs exploring different stages of magnetic field evolution in galaxies.
\end{abstract}

Key words: galaxies: magnetic fields - magnetic fields - polarization - quasars: absorption lines

\section{Introduction}

The evolution of magnetism in galaxies is of fundamental interest (Kronberg \& Perry 1982). In particular, the magnetic fields in young protogalaxies are largely unexplored (Wolfe et al. 1992; Oren \& Wolfe 1995). These protogalaxies are expected to have not yet experienced significant dynamo activity, and therefore constitute "missing links" in our understanding of the dynamo process and the evolution of cosmic magnetic fields (Gaensler et al. 2004). The dynamo process describes how weak "seed" magnets in the early universe were amplified and ordered throughout cosmological history by large-scale rotation and turbulence within galaxy disks and halos (e.g., Chamandy et al. 2014; Chamandy \& Taylor 2015). This suggests that the magnetic field strength should be weaker in protogalaxies than in normal galaxies. Furthermore, the coherence of the typical protogalactic magnetic field should also be less, with significant field disorder expected to be present. The strength of these magnetic fields has implications for the cosmological growth of magnetism and can constrain dynamo mechanisms (e.g., Beck et al. 2013). Such studies are highly challenging, as at radio wavelengths the protogalactic environment is typically of such low luminosity that directly imaging the emission due to magnetic fields will likely not even be possible with ultrasensitive (sub- $\mu \mathrm{Jy}$ ) data from the Square Kilometre Array (SKA; Johnston-Hollitt et al. 2015). We therefore suggest an alternative approach to studying magnetic fields in these galactic building blocks now, using radio polarization observations.

Radio polarization observations are the best available probe of cosmic magnetic fields, as they allow us to measure both the polarized fraction toward distant background radio sources (which is related to the degree of ordering of the intervening magnetic fields) and the Faraday rotation located along the entire line of sight. Faraday rotation is a powerful tool for measuring the magnetic field strength toward astrophysical objects. The combination of cosmic magnetic fields and charged particles distributed along the sightline toward a background radio source causes the rotation of the polarization angle of linearly polarized synchrotron emission (e.g., Longair 2011). Along a line of sight, the observed polarization angle is altered by an amount equal to

$$
\Phi=\Phi_{0}+\mathrm{RM} \lambda^{2},
$$

where $\lambda$ is the observing wavelength, $\Phi$ and $\Phi_{0}$ are the measured and intrinsic polarization angles respectively, and the constant of proportionality RM, the "rotation measure," is generally related to the integrated product of the electron number density, $n_{\mathrm{e}}$, and the strength of the component of the magnetic field parallel to the line of sight, $B_{\|}$. The observed $\mathrm{RM}$ is also related to the redshift at which the Faraday rotating medium is located, but as it is generally not known where all of the rotating media are distributed along the line of sight, this relation is typically not simple. Nevertheless, measurements of the RM can be used to infer the presence of magnetic fields somewhere along the line of sight between an observer and a source.

Using a quasi-stellar object (QSO, which we will use interchangeably with quasar) as a flashlight shining through and "back-lighting" foreground intervening material, it has previously been suggested that there is a correlation between metal-line absorption and the Faraday rotation/RM of distant 


\section{OBSERVER \\ FOREGROUND PROTOGALACTIC CLOUD}

BACKGROUND

POLARIZED RADIO

SOURCE

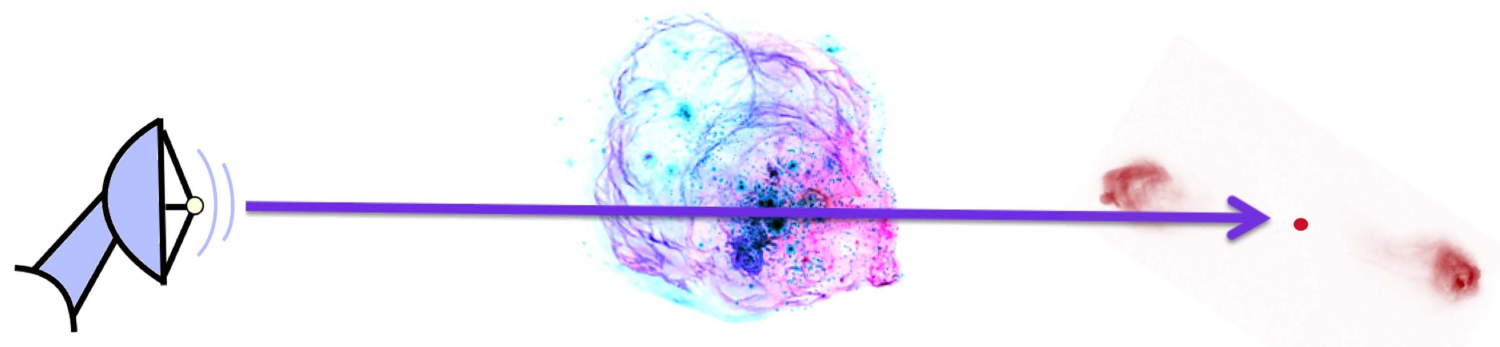

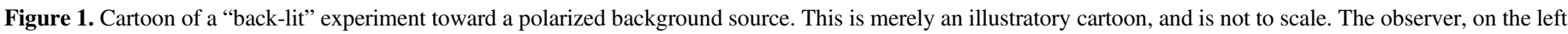

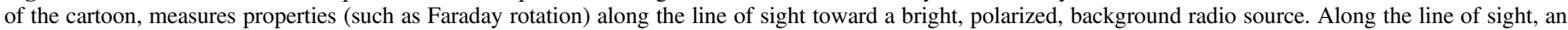

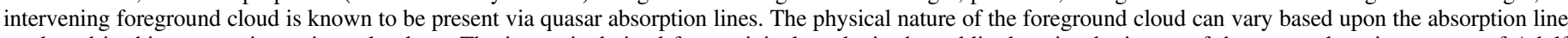

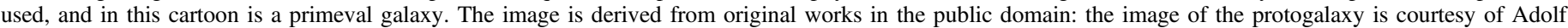
Schaller for STScI, and the image of Cygnus A is courtesy of NRAO/AUI/NSF.

polarized sources (e.g., Welter et al. 1984; Kronberg et al. 1992). The setup of a typical back-lit experiment is shown in Figure 1. Previous studies have already begun using the correlations seen in back-lit experiments to indirectly test dynamo theory in normal galaxies using strong magnesium II (Mg II) absorption lines (e.g., Bernet et al. 2008, 2013; Farnes et al. 2014b) located in the spectra of QSOs. This analysis can be extended to use Damped Lyman-Alpha Absorption systems and Lyman Limit Systems (LLSs) in the spectra of QSOs, and thereby probe the distribution of gaseous matter throughout the universe, particularly in the protogalactic and the intergalactic medium (IGM) (Rauch 1998; Wolfe et al. 2005; Meiksin 2009).

In this paper, we are interested in two types of absorption systems and define these systems via their neutral hydrogen column densities, with $1.6 \times 10^{17}<N(\mathrm{H} \mathrm{I})<2 \times 10^{20} \mathrm{~cm}^{-2}$ being LLSs and $N(\mathrm{HI}) \geqslant 2 \times 10^{20} \mathrm{~cm}^{-2}$ being Damped Lyman-Alpha Absorbers (DLAs; Wolfe et al. 2005; Erkal 2015). There is a fundamental difference between both systems: hydrogen is mainly neutral in DLAs, while it is mainly ionized in LLSs. This characteristic separates the DLAs from both the LLSs and other intervening absorbers seen in QSO sightlines, such as the Ly $\alpha$ forest $\left(N(\mathrm{H} \mathrm{I}) \leqslant 10^{17} \mathrm{~cm}^{-2}\right)$, where the neutral gas is a minor or non-existent phase. The presence of neutral, cold, and molecular gas is crucial to link the DLAs to star-forming galaxies (Ledoux et al. 2003; Wolfe et al. 2003, 2005; Howk et al. 2005; Noterdaeme et al. 2008). However, as we will discuss in Section 1.1, the exact nature of the DLAs and LLSs and their relation to present-day galaxies is still under debate and study, with numerous proposed origins (Wolfe et al. 2005; Wolfe \& Chen 2006; Rauch et al. 2008; Meiksin 2009). Although the nature of these absorption systems remains poorly understood, they possibly provide the only example of an interstellar medium (ISM) in the highredshift universe.

Both types of systems are important, as they are known to be some of the biggest intervening hydrogen gas reservoirs in the universe and thereby constitute the building blocks of galaxies. The combination of both DLAs and LLSs provides a large range in column density that allows us to explore and contrast the difference between these relative ends of the absorption system extrema. Importantly, both DLAs and LLSs are generally believed to correspond to similar features in the IGM. The column density of these absorbers has been found to be correlated to the mass of the nearest galaxy relative to the line of sight, with the correlation more pronounced for DLAs, and similar correlations found between the star formation rate, halo mass, and H I content of the associated galaxies (e.g., Rahmati \& Schaye 2014). Similarly, LLSs display a correlation between $N(\mathrm{HI})$ and halo mass, with lower column density systems more likely to be found near lower mass halos (e.g., van de Voort et al. 2012).

However, only two studies to date have attempted to look for connections between protogalaxies themselves and magnetic fields. The first study of Wolfe et al. (1992) used Mg II systems with H I column densities above $2 \times 10^{20} \mathrm{~cm}^{-2}$ and classified these as DLAs. They found five background sources with Faraday rotation higher than three times the $1 \sigma$ uncertainty in residual RM. The second study of Oren \& Wolfe (1995) used DLAs along the line of sight toward 11 background sources with known Faraday rotation. This data-limited study provided inconclusive results, with a "tentative" indication of higher Faraday rotation associated with the DLAs. Neither study included any LLSs in their sample. This is a similar scenario to the previously reported weak correlations between $|\mathrm{RM}|$ and the number of strong $\mathrm{Mg}$ II absorption lines along the line of sight (Kronberg \& Perry 1982; Bernet et al. 2012), which have more recently been expanded into definitive connections (Farnes et al. 2014b). The next step in understanding the magnetized structure, strength, and coherence in low-luminosity galaxies is therefore to expand from studies of $\mathrm{Mg}$ II absorption lines to new studies of DLAs and LLSs. We can attempt to use polarization measurements of background sources to measure the magnetic fields in the ionized gas component of these systems (e.g., Rubin et al. 2015).

\subsection{The Nature of DLAs and LLSS}

The fundamental nature of both DLAs and LLSs is uncertain. There have been several suggestions as to the physics and origin of these systems, often with varying degrees of certainty. Consequently, the exact nature of the DLAs and LLSs and their relation to present-day galaxies is still under debate and study, with numerous proposed origins. Previous studies have typically been limited by low sample sizes, uncertain statistical significance, and complicated interpretation of both statistical results (e.g., $p$-values, often on the order of $p=0.05$ ) and of the physics (e.g., different galaxy types and 
evolving populations as a function of redshift). There is some belief that these systems are believed to probe the progenitors of current massive galaxies (e.g., Songaila \& Cowie 2010), with DLAs corresponding to extended disks (e.g., Prochaska \& Wolfe 1998) and LLSs corresponding to extended gaseous halos (e.g., Erkal 2015). Nevertheless, in this section we present the various findings and interpretations within the contemporary literature, and the emerging consensus that both DLAs and LLSs at $z \approx 2$ have a relationship to protogalactic systems.

While there is an emerging body of evidence that DLAs and LLSs definitively probe the distribution of collapsed, cold gas at high redshift and at different H I column densities (e.g., Gardner et al. 1997), any related distinctions between the probed environments or galaxy types are still a matter of debate. Suggestions include that DLAs and LLSs may be related to different galaxy components such as disks versus halos, to high- versus low-impact parameters, or to clouds of turbulent halo gas versus more quiescent gas (Steidel 1993; Meylan 1995; Rao et al. 2011). It has also been argued that photometry of higher column density DLAs lends support to the view that those systems are high-redshift galaxies (Djorgovski et al. 1996; Fontana et al. 1996). Meanwhile, studies of lower column density systems similar to the LLSs indicate that those systems can even be associated with lines of sight that pass near galaxy groups or clusters (Lanzetta et al. 1996). It is of interest to investigate the connection between DLAs and LLSs, in order to better understand the links with galaxy formation. However, it is strongly likely that both systems provide insight into the nature of protogalaxies in the early universe (Prochaska 1999; Wolfe et al. 2005).

To date, our understanding of DLAs and LLSs has mostly been developed through observations, rather than through theory. This has arguably resulted in a relatively simple model of the physics that drives the evolution and growth of these objects, and resulted, for example, in possible abundance mismatches between simulations and observations (Gardner et al. 2001). Nevertheless, the consistent consensus is that DLAs and LLSs are overdense regions in the IGM, and are therefore protogalactic clumps of some form (e.g., Prochaska 1999; Wolfe et al. 2005). Such systems likely constitute the predecessors of modern galaxies and have been proposed to be the progenitors of massive elliptical galaxies (Prochaska et al. 2003) and normal disk galaxies (Djorgovski et al. 1996). As such systems constitute significant neutral gas reservoirs and overdensities in the universe, we therefore know that these systems must be connected to general galactic evolution.

There is considerable evidence that DLAs are related to protogalaxies (Wolfe \& Prochaska 2000). More contemporary work (e.g., Rubin et al. 2015) has also indicated that some fraction of DLAs occur in cold and dense inflowing streams (that extend over tens of kiloparsecs) that feed star formation in a massive central galaxy (Razoumov et al. 2008; Fumagalli et al. 2011; Cen 2012). There is also some evidence that DLAs can trace wind material that has been lifted away from galactic disks by star formation-driven outflows (Pontzen et al. 2008; Razoumov et al. 2008).

There is also a body of evidence that suggests LLSs probe at least three different system types. It has been argued that the LLSs are associated with systems similar to the DLAs (Prochaska 1999) and with the halos of low-mass galaxies (Erkal 2015). They are also known to trace infall onto galaxies, being consistent with cold mode accretion streams (Ribaudo et al. 2011; Fumagalli et al. 2016). The same mechanism is also believed to be responsible for some DLAs (Fumagalli et al. 2011). The LLSs have also been suggested to trace outflows, including those driven by star formation and AGN activity, but also potentially including Galactic superwinds (Prochaska et al. 2006; Tripp et al. 2011). As LLSs are clearly seen to trace phenomena that are associated with the circulation of matter into and out of galaxies, and as LLSs form a "bridge" between the higher column density DLAs and the lower column density Ly $\alpha$ forest, it is possible that LLSs may also trace the interface between protogalaxies and the IGM. This is supported by numerical simulations that find that LLSs occur on lines of sight that pass through the outer parts of more massive protogalaxies (and also near the center of younger, lower density systems; Katz et al. 1996). Either way, the LLSs are potentially associated with the medium surrounding galaxies, e.g., the circumgalactic medium.

Consequently, DLAs and LLSs offer potential probes to trace the evolution of these various phenomena throughout cosmic time. Furthermore, the study of protogalactic clumps, and consequent galactic building blocks, potentially opens a window for studying magnetic fields in the cosmic web. As both systems are known to have some relation to infall and outflows, there is also an overlap with the more well-studied Mg II systems (e.g., Steidel \& Sargent 1992; Steidel et al. 1997; Kacprzak et al. 2007; Mshar et al. 2007). There is even some evidence to suggest that strong Mg II systems and LLSs probe similar physical systems (Steidel 1993; Churchill et al. 1999), and consequently LLSs would extend the measurement of strong Mg II systems out to higher redshifts. Nevertheless, while this is difficult to reconcile with the very different column densities probed by such systems, it is likely that these various absorption line systems have considerable overlap and can probe a range of different environments. However, via QSO-absorption line studies, we can still study the average system represented at each respective column density range. It is likely that a considerable leap in sample size would be required, in order to clearly identify the overlaps between the physical systems measured at different column densities (and also to avoid any arbitrary distinctions based on column density). This is especially true as each physical system also likely evolves with redshift and other parameters, which ideally require isolation.

This paper is structured as follows: we present our observational data in Section 2, which details and justifies how we created our sample and its properties. Our results, including a quantitative analysis of our main sample, are detailed in Section 3. A discussion of our results and the estimation of magnetic properties from our data is presented in Section 4, while our conclusions are summarized in Section 5. We refer to "polarization" on multiple occasions-in all cases we are referring to linear radio polarization. Unless otherwise specified, all quantities are presented as measured in the observed frame.

\section{Sample Construction}

To create the sample, we have concatenated various DLA and LLS identifications taken from across the literature. A summary flowchart for the production of our final samples is shown in Figure 2. As our initial starting catalog, we have used the ninth data release of the Sloan Digital Sky Survey (SDSS) 


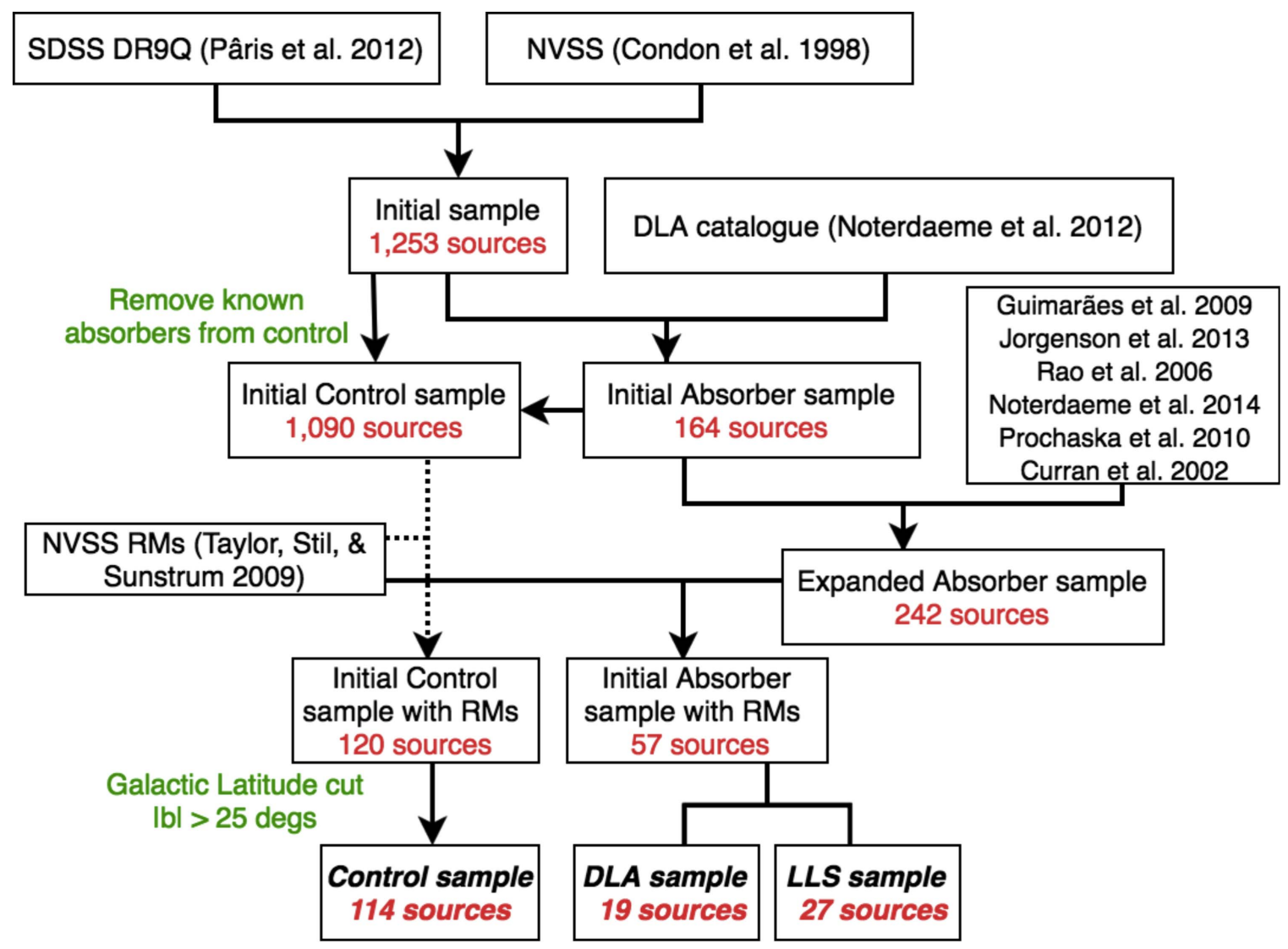

Figure 2. Flowchart showing the production of our final control, DLA, and LLS samples. Multiple catalogs were used to produce the final data set.

Quasar Catalog, DR9Q, of 87,822 sources (Pâris et al. 2012). We have cross-matched this catalog to the original NRAO Very Large Array Sky Survey (NVSS; Condon et al. 1998). In order to only incorporate those sources with the most definitive, single-component, compact optical and radio counterparts, we use a 2 arcsec cross-matching radius. For the astrometry, we are here not concerned with systematics in the NVSS: sources with detectable polarization are bright in total intensity and typically have accurate positions to $<1$ arcsec (Farnes et al. 2014a). The tight cross-matching radius is instead essential given previous experience with back-lit absorption line experiments, as we need to match the same source components and corresponding lines of sight through the universe (Farnes et al. 2014b). This is typically challenging given the different radio and optical source morphologies, which can sample lobes and cores, respectively. However, a sampling of cores can be trivially achieved to first order via a tight cross-matching radius. This yields 1253 sources and is our initial sample. We have then used a DLA catalog of 12,081 quasars that were observed in the course of the Baryon Oscillation Spectroscopic Survey (BOSS), which is also part of the SDSS DR9 (Noterdaeme et al. 2012). Each of the quasars has one detected intervening DLA or LLS along the line of sight. We have also crossmatched this catalog to the original NVSS with a 2 arcsec radius. This yields 164 sources and is our initial absorber sample. Both the radio-matched control catalog of 1253 sources (Pâris et al. 2012) and also the radio-matched DLA/LLS catalog of 164 sources (Noterdaeme et al. 2012) were derived using the same data set. In Noterdaeme et al. (2012), all sources from Pâris et al. (2012) were searched for DLAs/LLSs, and only those with positive detections were listed. Further details of the criteria used to identify DLAs/ LLSs are provided in Noterdaeme et al. (2009, 2012). Consequently, we therefore remove sources from the initial sample in which DLAs/LLSs were positively identified. This reduces the initial sample into an initial control sample of 1090 sources in which no intervening DLAs or LLSs were identified. We then proceed to incorporate various other DLA catalogs gathered from throughout the literature, and which we again pre-match with the original NVSS using a 2 arcsec radius. The sample of Guimarães et al. (2009) provides two sources, Jorgenson et al. (2013) provides five sources, Rao et al. (2006) provides 43 sources, Noterdaeme et al. (2014) provides two sources, Prochaska et al. (2010) provides 50 sources, and Curran et al. (2002) provides 39 sources. We also attempted to include data from Turnshek et al. (2015), but identified no NVSS radio counterparts. This yielded an expanded absorber sample of 242 sources.

In order to check whether there were any duplicated matches, we cross-checked these additional data against the initially formed control and DLA/LLS samples. Of the five sources from Jorgenson et al. (2013), two sources were found to have existing matches in the DLA/LLS sample, with manual inspection showing consistent estimates for the redshift of the absorber and the neutral hydrogen column density. As the data of Jorgenson et al. (2013) were derived from SDSS DR5, we instead propagated the DR9 match into the final absorber catalog. The three additional sources without existing records were also added into the sample of absorbers. Similarly, of the two sources from Noterdaeme et al. (2014), a single source was found to have an existing match in the DLA/LLS sample. As the sources from Noterdaeme et al. (2014) are from SDSS 
DR11, the existing record for this one data point was replaced. However, manual inspection again showed that the redshift of the absorber and the neutral hydrogen column density of both data were in agreement within their uncertainties. The one additional source without an existing record was added into the sample. Finally, of the 39 sources from Curran et al. (2002), a single source was found to have an existing match in the control sample and a further nine sources were found to have existing matches in the DLA/LLS sample. As Curran et al. (2002) use older data, we rely on the accuracy of the Pâris et al. (2012) data and discard the data of the single source with a Curran et al. (2002) match. For the nine sources in the DLA/LLS sample, manual inspection showed that for seven sources the redshift of the absorber and the neutral hydrogen column density of both data were in agreement within their uncertainties. Nevertheless, two sources have updated estimates of source parameters in the contemporary data, implying either a $\sim 7 / 9=77 \%$ accuracy rate in the earlier measurements, or possibly a systematic affecting the newer data. However, both the old and new data are in agreement that these sightlines do contain intervening DLAs/LLSs. We therefore keep our current estimates and do not update our catalog values using these nine sources. The remaining 29 sources are added into the DLA/LLS sample, with an awareness that $\approx 6$ of these sources may not have accurate estimates of the source parameters. For some sources, we cannot rule out, e.g., low-redshift intervenors observable in the ultraviolet part of the spectrum, due to the spectral coverage provided. However, there is no reason to expect any differences between the control, DLA, and LLS samples. Effectively, all of our samples should contain lowredshift intervenors to a similar degree, and so this cannot explain any $|\mathrm{RM}|$ enhancement that we might detect. While the source parameters (redshifts and column densities) may not be fully accurate, this extra sample still contains information that can improve our analysis. Although the redshifts and column densities of these sources are in dispute, both the old and new sets of data are consistent in their classification that these sources do contain intervening DLAs/LLSs. Our classification is therefore reliable. As we wish to obtain a statistical detection of magnetic fields in different galaxy classifications, this is the key knowledge that is required for each included line of sight.

We have created an initial control sample of 1090 sources with no known DLAs/LLSs, and an extended absorber sample of 242 sources with an intervening DLA/LLS along the line of sight. From cross-matching the catalog of Curran et al. (2002), we found that $\approx 6$ sources may not have accurate source parameters, indicating that $\leqslant 6 / 242=2.5 \%$ of sources have not been accurately classified. Nevertheless, as this only affects our oldest catalog, the classification accuracy is likely considerably better. Each quasar has both an optical detection and a $1.4 \mathrm{GHz}$ radio counterpart. As all data were crossmatched using a very tight 2 arcsec cross-matching radius, we have only included the most definitive matches into our sample. This should alleviate the need to split our sample by spectral index as a proxy for enhanced resolution, as has been done in studies of strong Mg II absorption (Farnes et al. 2014b). This has been essential for Mg II studies, in order to ensure that the same optical and radio line of sight through the universe is measured. Whereas the optical emission tends to sample the core of an AGN, the radio emission tends to sample the lobes (with the lobes preferentially sampled at increasingly lower

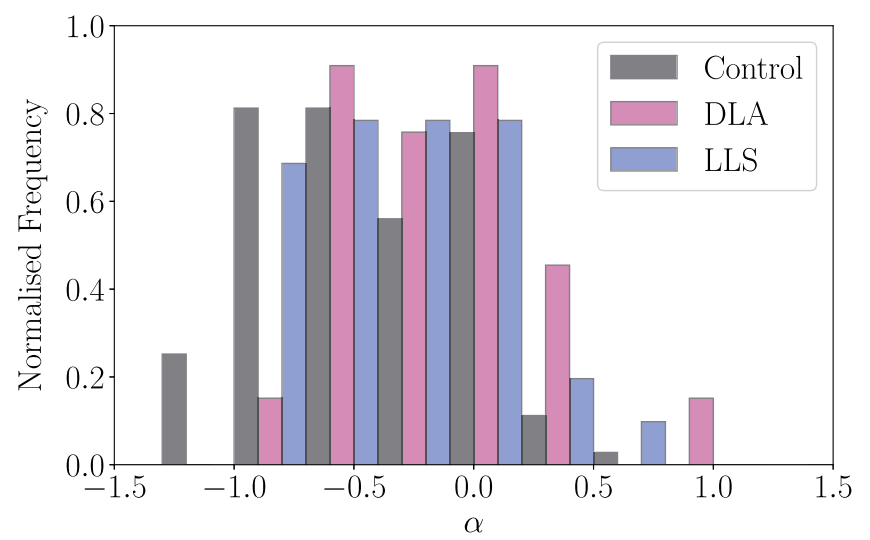

Figure 3. Spectral index distribution for our sample. Lines of sight categorized into the control sample (black), the DLA sample (violet), and the LLS sample (blue) are all shown in the normalized histogram.

frequencies, due to the lobes' steep spectral index). Due to this effect, radio sources can be cross-matched to optical/ultraviolet sources with increased precision by only matching flatspectrum sources. However, unlike strong Mg II, which is associated with a clumpy partially ionized medium, DLAs and LLSs are associated with a smooth medium with high covering fraction (see Section 4). The spectral index criterion is therefore likely not an essential requirement for DLAs/LLSs (although we lack the sample size to thoroughly test this hypothesis). The spectral index distribution of the final sample of sources is shown in Figure 3.

As we are here chiefly interested in the radio polarization, we continue to cross-match our samples with the NVSS catalog of Taylor et al. (2009), in which all sources are polarized at $\geqslant 8 \sigma$. This high signal-to-noise ratio $(\mathrm{S} / \mathrm{N})$ detection threshold is crucial so that Rician bias effects are negligible (Simmons \& Stewart 1985). Rician bias occurs as the noise in polarized intensity is positive-definite, so that even in the absence of signal, one obtains a non-zero measurement. This leads to an overestimate of all polarized intensity measurements, with the magnitude of the effect decreasing for brighter sources. If Rician bias effects were significant, then our experimental design would be fundamentally flawed. Fainter sources have a larger bias in fractional polarization, and so these sources would appear more polarized than they truly are. To counter this effect, we would need to check that all of the samples cover similar distributions of polarized and total intensity. However, this would not be possible, as one of our primary science questions is whether the samples have different polarized fractions - which depends on different distributions of polarized and total intensity. Ultimately, this would result in a circular argument. For the cross-matching, we use a 2 arcsec cross-matching radius to combine the Taylor et al. (2009) and the Condon et al. (1998) versions of the NVSS. We also use the original NVSS catalog in order to calculate the effective area of each source, using $A=\frac{1}{4} \pi \theta_{M} \theta_{m}$, where $\theta_{M / m}$ are the major and minor axes, respectively. The effective area distribution of the final sample of sources is shown in Figure 4. For the NVSS's $45 \mathrm{arcsec}$ beam, the effective area is $1590 \mathrm{arcsec}^{2}$. This suggests that the sources are all smaller than the beam size. Further inspection of the NVSS data highlights that the listed sizes are in fact upper limits: all of these sources are compact radio sources at NVSS resolution at $1.4 \mathrm{GHz}$. This is 


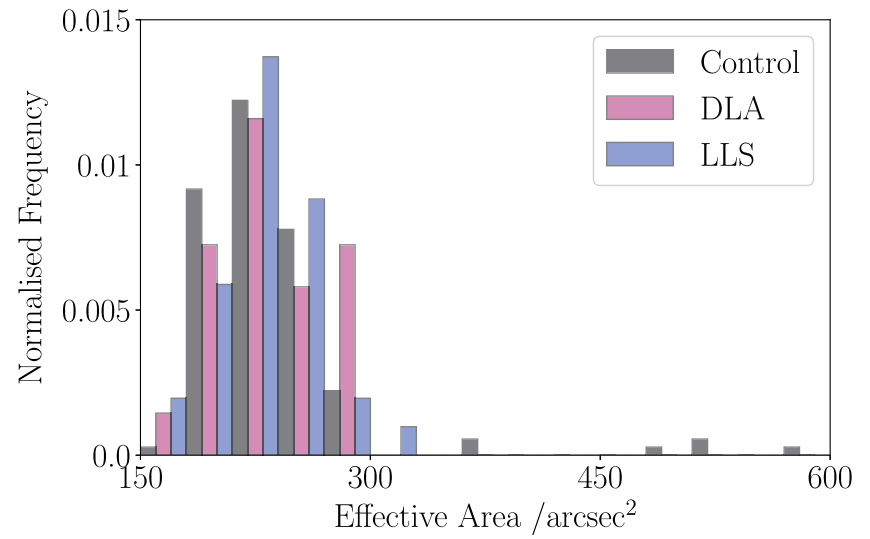

Figure 4. Distribution of the effective area for each source in our sample. Lines of sight categorized into the control sample (black), the DLA sample (violet), and the LLS sample (blue) are all shown in the normalized histogram.

unsurprising given our tight cross-matching criterion, which is designed to select only polarized compact radio sources at NVSS resolution. The effective area distribution therefore demonstrates that our cross-matching procedure has been successful. We would ideally like to restrict the sample to sources that could reasonably have the polarized flux passing through the extended absorbing region that has been sampled by the QSO. This can be achieved in two ways. (i) By measuring the polarized source sizes directly, although this is not possible with the current data. However, the fact that all of our sources are smaller or on the order of the beam size at the NVSS resolution of 45 arcsec helps considerably. The polarized intensity cannot be strongly offset from the total intensity position. DLAs are typically expected to have diameters of $\sim 15 \mathrm{kpc}$ whereas LLSs are typically expected to have diameters of $\sim 80 \mathrm{kpc}$ (Churchill et al. 1999), which at $z=2$ in a $\Lambda C D M$ cosmology corresponds to 1.8 arcsec and 9.3 arcsec respectively. Our cross-matching radius accesses these size scales. (ii) Restricting source sizes by use of the spectral index, since flat-spectrum sources will be dominated by a compact, often parsec-scale core coincident within the QSO. However, there are many small steep-spectrum sources that would be excluded from the sample, but would still be backlighting the absorber. Due to the large decrease in sample size using this method, we rely on the source size measurements. It is possible, with future data, that one could produce cleaner samples based on background source size and structure that would more directly probe the absorbing region and further improve the robustness of the results.

Some of our catalogs have varying and inconsistent definitions of the column densities associated with DLAs and LLSs, and so we re-sort the data such that systems with $1.6 \times 10^{17}<N(\mathrm{HI})<2 \times 10^{20} \mathrm{~cm}^{-2}$ are classified as LLSs and $N(\mathrm{HI}) \geqslant 2 \times 10^{20} \mathrm{~cm}^{-2}$ are classified as DLAs (e.g., Erkal 2015). The column density distribution of the final sample of sources is shown in Figure 5.

We have now obtained an initial control sample with RMs of 120 sources and an initial absorber sample with RMs of 57 sources (23 DLAs and 34 LLSs) - a total of 177 sources. However, in order to remove the effects of the Galactic foreground, we also now exclude sources located at Galactic latitudes $|b| \leqslant 25^{\circ}$. This yields a control sample of 114 sources, a DLA sample of 19 sources, and an LLS sample of 27 sources - a total of 160 sources. All of these sources have known radio

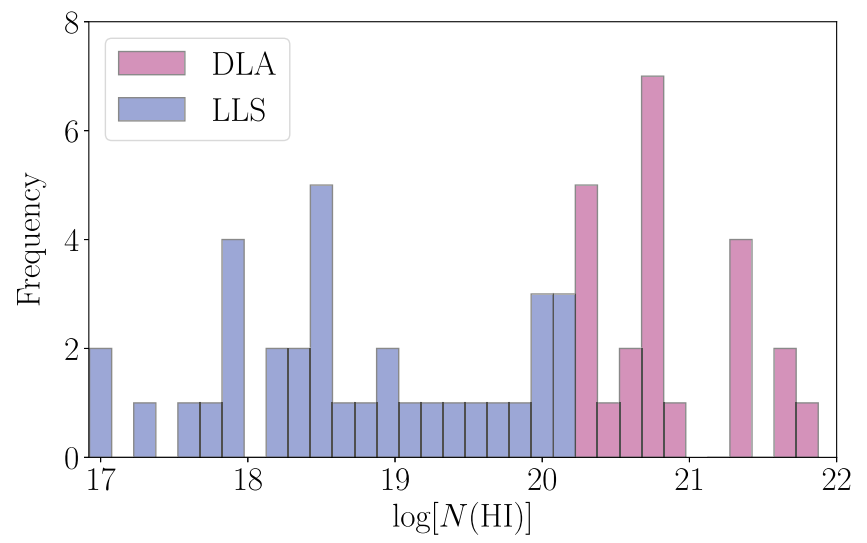

Figure 5. Column density distribution for our samples. Lines of sight categorized into the DLA sample (violet) and the LLS sample (blue) are shown in the histograms.

polarization fractions and RMs from Taylor et al. (2009). Note that this final updated sample has five times more Lymanabsorbing systems than the largest published study in this area (Oren \& Wolfe 1995), which even then did not include any LLSs. Our sample includes ultraviolet and optically identified DLAs/LLSs - and covers a significant range in redshift space with absorbers from $0.23 \leqslant z \leqslant 3.81$. The redshift distribution of the final sample of sources is shown in Figure 6. The properties of each source used in our analysis are given in Table 1. A statistical overview of our data set is given in Table 2 .

\section{Results}

\subsection{The Data}

In principle, it is possible to remove the Faraday rotation contribution from the Galactic foreground (GRM) directly by subtracting this from the RM, in order to obtain a Residual Rotation Measure (RRM), using $\mathrm{RRM}=\mathrm{RM}-\mathrm{GRM}$. The RRM should consist of the extragalactic contribution to the lineof-sight Faraday rotation for each source. However, in practice, with current restrictions the RRM cannot be reliably calculated as it contain systematics from the foreground calculation alongside an additional inherent error term (see Oppermann et al. 2015). To quote Oppermann et al. (2015, p. 19), "extragalactic contributions are not very well constrained by the data" and "subtracting a Galactic foreground from the data is therefore not a good way of estimating extragalactic contributions." This inhibits analysis of the RRMs, unless some additional technique can be invoked to account for the extra uncertainties (see Section 5). With the current RM grid, this makes analysis of the GRMs a more reliable approach (as discussed further in Section 3.2). For our analysis, we use the GRM data from Oppermann et al. (2015).

Our RM and GRM data are presented in Figure 7. Our polarized fraction, $\Pi=\sqrt{Q^{2}+U^{2}} / I$, data are presented in Figure 8. The figures show all three of our individual samples: the control, DLA, and LLS. The distributions of the various samples cannot be assessed reliably by eye, due to the small number statistics. We now will assess statistical differences between each of the samples using a full Bayesian framework. A Bayesian analysis is extremely advantageous for statistical analysis with low sample sizes, such as those often encountered 

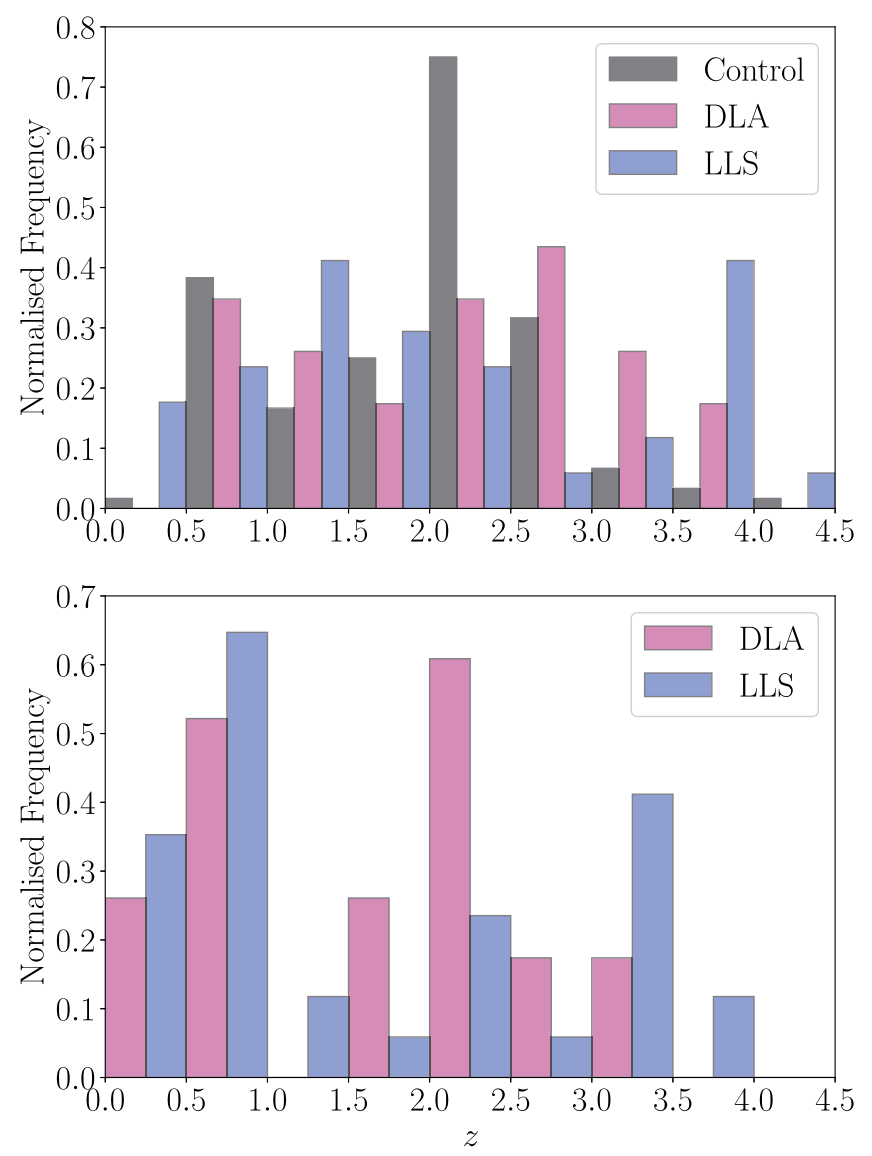

Figure 6. Redshift distributions for our sample. Lines of sight categorized into the control sample (black), the DLA sample (violet), and the LLS sample (blue) are all shown in the normalized histograms. Top: the redshift distribution of the background QSOs. Bottom: the redshift distribution of the absorbing systems.

in astronomy, as the analysis of small data sets can otherwise lead to statistical power issues and often suffer from biased parameter values. Similarly, in the "Big Data" era, fully Bayesian tests can avoid finding significant correlations in cases where none exist. A full introduction to Bayesian analysis is well beyond the scope of this paper, and we refer the reader to the classic text of Gelman et al. (2004) for extensive further details on the conventions of the method and notation. Extensive details on the Bayesian framework that we use, including the selection of our priors, is provided in the Appendix.

The analysis of the Faraday rotation (RMs and GRMs) is given in Section 3.2, while the analysis of the polarized fractions is given in Section 3.3. For our parameter estimates of the distribution of Faraday rotation, we estimate the parameters $\mu$ and $\sigma$, which correspond to the calculated mean and standard deviation of the Faraday rotation of our source population (which we model using a normal distribution). For our parameter estimates of the polarized fractions, we estimate the parameters $\mathrm{E}[\Pi]$ and $\mathrm{SD}[\Pi]$, which correspond to the arithmetic mean and arithmetic standard deviation of the polarized fractions of our source population (which we model using a log-normal distribution). In both cases, we estimate these parameters from the posterior using the 0.5 quantile, i.e., the median, and define the specified uncertainties with the $68 \%$ credible interval. For extensive further details, please refer to the Appendix. On multiple occasions, we will refer to the typical RM and polarized fraction of each sample. As Faraday rotation can be both positive and negative, $\mu$ tends to be centered at $0.0 \mathrm{rad} \mathrm{m}^{-2}$. However, we are interested in $|\mathrm{RM}|$ (and $|\mathrm{GRM}|$ ) rather than $\mathrm{RM}$, and this quantity is best parameterized by $\sigma$, which informs us of the typical magnitude of RM in our distribution. As we here allow a sign dependence in the distribution of RMs, this is equivalent to the $|R M|$ used in previous studies. The typical polarized fraction is parameterized by $\mathrm{E}[\Pi]$, which informs us of the typical magnitude of the polarized fraction for each sample.

\subsection{Faraday Rotation}

We first consider the case of the RM and GRM values associated with the DLA and LLS. Without removal of the Galactic foreground, for a sample of sources that is evenly distributed spatially across the sky, we select a normal (Gaussian) distribution as our model for the RMs (e.g., Hammond et al. 2012; Farnes et al. 2014a). However, a sample of sources that is not evenly distributed on the sky is expected to have a non-Gaussian distribution of RMs due to large-scale magnetic fields in the Milky Way that become imprinted onto the Faraday rotation signal of the background sources. It is therefore important to test that the Galactic foreground does not vary substantially between different samples. This Galactic foreground has been estimated in various studies (Taylor et al. 2009; Oppermann et al. 2012; Johnston-Hollitt et al. 2015; Oppermann et al. 2015) and can in principle be subtracted from the RM signal to yield an RRM (as discussed in Section 2). Nevertheless, it has been suggested that while the Galactic foreground can be estimated using surveys such as the NVSS, it cannot be reliably subtracted to obtain an RRM without knowing the relative uncertainties (Oppermann et al. 2015). We therefore check for Galactic variations using the GRMs, which are free from contamination by additional uncertainties. This methodology was also used in Farnes et al. (2014b) and is akin to stating that $\sigma_{\mathrm{RM}}^{2}=\sigma_{\mathrm{GRM}}^{2}+\sigma_{\mathrm{RRM}}^{2}$. The sampled posterior distributions for the RMs and GRMs are shown in Figure 9. It is clear from the posterior distributions that the results differ substantially from the prior distribution. The data dominate our result.

To answer our scientific question, we want to know: what is the probability that the RMs of the control sample are smaller than the RMs of the DLA (or LLS) sample, given our data? Or equivalently, for the DLAs,

$$
p(\sigma[\mathrm{DLA}]>\sigma[\text { control }] \mid D)
$$

where the probability is an evidential or Bayesian probability, rather than a frequentist probability.

We evaluate Equation (2), for the controls, DLAs, and LLSs through numerical integration by counting the number of posterior samples that satisfy, e.g., $\sigma$ [DLA] $>\sigma$ [control], and dividing by the total number of samples $(2,000,000)$ drawn. The obtained probabilities are given in Table 3 .

\subsection{Polarized Fractions}

The sampled posterior distributions for the polarized fractions, $\Pi$, are shown in Figure 10. It is again clear from the posterior distributions that the results differ substantially from the prior distribution. The data dominate these results. 
Table 1

Source Parameters for All of the Polarized QSOs Used in our Analysis

\begin{tabular}{|c|c|c|c|c|c|c|c|c|c|}
\hline $\begin{array}{l}\text { R.A. } \\
\text { (degrees) }\end{array}$ & $\begin{array}{c}\text { Decl. } \\
\text { (degrees) }\end{array}$ & $\begin{array}{c}\mathrm{RM} \\
\left(\operatorname{rad~m^{-2}}\right)\end{array}$ & $\begin{array}{c}\text { GRM } \\
\left(\operatorname{rad~m}^{-2}\right)\end{array}$ & $\begin{array}{c}\Pi \\
(\%)\end{array}$ & $\alpha$ & $z_{\mathrm{qso}}$ & $z_{\mathrm{abs}}$ & $\log \left[N(\mathrm{H} \mathrm{I}) / \mathrm{cm}^{-2}\right]$ & $\begin{array}{c}\text { Type } \\
(\mathrm{C} / \mathrm{D} / \mathrm{L})\end{array}$ \\
\hline 5.901 & -2.885 & -29.1 & -3.4 & 4.05 & -0.70 & 2.74 & $\ldots$ & $\ldots$ & $\mathrm{C}$ \\
\hline 16.596 & -1.927 & -9.5 & 1.6 & 7.62 & -0.33 & 2.19 & $\ldots$ & $\ldots$ & $\mathrm{C}$ \\
\hline 17.112 & -0.623 & 6.2 & 6.6 & 6.91 & -0.31 & 1.38 & $\ldots$ & $\ldots$ & $\mathrm{C}$ \\
\hline 22.752 & 0.290 & 15.5 & 13.5 & 8.95 & -1.07 & 4.02 & $\ldots$ & $\ldots$ & $\mathrm{C}$ \\
\hline 23.652 & 6.210 & -1.7 & 3.3 & 4.32 & -0.96 & 1.00 & $\ldots$ & $\ldots$ & $\mathrm{C}$ \\
\hline 25.080 & 14.774 & -12.2 & -9.6 & 11.82 & -0.47 & 2.23 & $\ldots$ & $\ldots$ & $\mathrm{C}$ \\
\hline 27.343 & 5.931 & 28.8 & 9.4 & 0.91 & 0.26 & 2.35 & $\ldots$ & $\ldots$ & $\mathrm{C}$ \\
\hline 115.482 & 42.138 & -0.9 & 1.1 & 5.02 & -0.65 & 2.23 & $\ldots$ & $\ldots$ & $\mathrm{C}$ \\
\hline 115.656 & 39.743 & 0.3 & 2.6 & 3.15 & -0.15 & 2.20 & $\ldots$ & $\ldots$ & $\mathrm{C}$ \\
\hline 117.122 & 50.888 & -9.4 & -1.7 & 1.82 & -0.95 & 0.63 & $\ldots$ & $\ldots$ & $\mathrm{C}$ \\
\hline 120.067 & 53.672 & -47.0 & -8.0 & 12.34 & -0.56 & 1.93 & $\ldots$ & $\ldots$ & $\mathrm{C}$ \\
\hline 120.228 & 45.014 & 9.9 & 11.2 & 9.12 & -0.42 & 2.36 & $\ldots$ & $\ldots$ & $\mathrm{C}$ \\
\hline 121.243 & 49.813 & -6.6 & -3.3 & 6.73 & -0.57 & 2.96 & $\ldots$ & $\ldots$ & $\mathrm{C}$ \\
\hline 121.640 & 45.075 & 23.9 & 20.7 & 2.24 & 0.14 & 2.11 & $\ldots$ & $\ldots$ & $\mathrm{C}$ \\
\hline 121.954 & 45.264 & 33.3 & 21.3 & 4.65 & -0.52 & 3.19 & $\ldots$ & $\ldots$ & $\mathrm{C}$ \\
\hline 122.905 & 40.789 & 17.5 & 22.7 & 5.85 & -0.79 & 0.67 & $\ldots$ & $\ldots$ & $\mathrm{C}$ \\
\hline 123.448 & 53.798 & -19.9 & -6.4 & 4.24 & -0.52 & 0.95 & $\ldots$ & $\ldots$ & $\mathrm{C}$ \\
\hline 123.599 & 19.984 & -15.3 & 4.7 & 5.61 & -0.86 & 1.50 & $\ldots$ & $\ldots$ & $\mathrm{C}$ \\
\hline 123.893 & 33.091 & 2.6 & 5.9 & 2.02 & -0.88 & 2.42 & $\ldots$ & $\ldots$ & $\mathrm{C}$ \\
\hline 124.263 & 16.383 & 42.5 & 38.4 & 4.59 & -1.00 & 0.70 & $\ldots$ & $\ldots$ & $\mathrm{C}$ \\
\hline 125.921 & 29.475 & 9.6 & 11.9 & 2.02 & -0.03 & 2.37 & $\ldots$ & $\ldots$ & $\mathrm{C}$ \\
\hline 126.125 & 31.726 & -3.7 & 25.2 & 9.96 & -1.11 & 2.37 & $\ldots$ & $\ldots$ & $\mathrm{C}$ \\
\hline 126.515 & 52.795 & -29.3 & -10.0 & 5.15 & -0.41 & 1.53 & $\ldots$ & $\ldots$ & $\mathrm{C}$ \\
\hline 126.519 & 36.115 & 26.2 & 20.3 & 3.16 & -0.69 & 1.03 & $\ldots$ & $\ldots$ & $\mathrm{C}$ \\
\hline 126.778 & 10.873 & 19.1 & 20.6 & 6.38 & -0.16 & 2.28 & $\cdots$ & $\cdots$ & $\mathrm{C}$ \\
\hline 127.039 & 29.339 & 18.2 & 19.7 & 1.76 & -0.51 & 2.32 & $\ldots$ & $\ldots$ & $\mathrm{C}$ \\
\hline 128.206 & 15.902 & 14.3 & 15.3 & 1.27 & -0.06 & 2.42 & $\ldots$ & $\ldots$ & $\mathrm{C}$ \\
\hline 128.310 & 11.393 & 52.8 & 40.9 & 1.49 & -0.26 & 2.98 & $\ldots$ & $\ldots$ & $\mathrm{C}$ \\
\hline 129.095 & 27.481 & 24.0 & 17.4 & 1.67 & -0.10 & 0.76 & $\ldots$ & $\ldots$ & $\mathrm{C}$ \\
\hline 129.205 & 11.784 & 26.9 & 22.2 & 4.8 & $\ldots$ & 2.41 & $\ldots$ & $\ldots$ & $\mathrm{C}$ \\
\hline 131.121 & 38.515 & 9.2 & 15.2 & 1.02 & -0.35 & 2.94 & $\ldots$ & $\ldots$ & $\mathrm{C}$ \\
\hline 136.192 & 2.145 & -3.6 & -4.4 & 4.12 & -0.65 & 0.79 & $\ldots$ & $\ldots$ & $\mathrm{C}$ \\
\hline 137.292 & 1.360 & -18.0 & -14.4 & 6.22 & 0.17 & 1.02 & $\ldots$ & $\ldots$ & $\mathrm{C}$ \\
\hline 137.316 & 3.912 & 38.0 & 35.6 & 5.4 & 0.02 & 3.29 & $\ldots$ & $\ldots$ & $\mathrm{C}$ \\
\hline 137.920 & 2.088 & -12.2 & -2.4 & 3.25 & -0.01 & 2.37 & $\ldots$ & $\ldots$ & $\mathrm{C}$ \\
\hline 143.811 & 1.537 & -38.0 & -16.4 & 4.78 & -0.80 & 2.55 & $\ldots$ & $\ldots$ & $\mathrm{C}$ \\
\hline 143.882 & 36.555 & -5.3 & 14.3 & 1.5 & 0.11 & 2.85 & $\ldots$ & $\ldots$ & $\mathrm{C}$ \\
\hline 149.512 & 38.500 & -33.8 & 7.5 & 1.81 & -0.97 & 1.40 & $\ldots$ & $\ldots$ & $\mathrm{C}$ \\
\hline 154.546 & 35.711 & 6.4 & 7.1 & 2.88 & 0.07 & 1.23 & $\ldots$ & $\ldots$ & $\mathrm{C}$ \\
\hline 154.920 & 4.733 & -7.0 & 10.1 & 10.83 & -0.23 & 2.29 & $\ldots$ & $\ldots$ & $\mathrm{C}$ \\
\hline 156.249 & 6.415 & 68.7 & 21.3 & 1.45 & -1.01 & 1.74 & $\ldots$ & $\ldots$ & $\mathrm{C}$ \\
\hline 156.633 & 6.459 & 29.7 & 21.3 & 1.6 & -0.86 & 2.46 & $\ldots$ & $\ldots$ & $\mathrm{C}$ \\
\hline 157.120 & 35.885 & -12.5 & 5.6 & 3.51 & -0.42 & 2.60 & $\ldots$ & $\ldots$ & $\mathrm{C}$ \\
\hline 160.175 & 36.903 & 22.5 & 11.0 & 8.24 & -0.68 & 2.69 & $\ldots$ & $\ldots$ & $\mathrm{C}$ \\
\hline 161.044 & 35.153 & 1.0 & 16.9 & 4.9 & -0.88 & 2.22 & $\ldots$ & $\ldots$ & $\mathrm{C}$ \\
\hline 163.632 & 38.923 & 18.5 & 9.5 & 7.51 & -0.16 & 1.37 & $\ldots$ & $\ldots$ & $\mathrm{C}$ \\
\hline 165.932 & 41.219 & 28.8 & 12.2 & 2.07 & -0.61 & 2.46 & $\ldots$ & $\ldots$ & $\mathrm{C}$ \\
\hline 168.161 & 34.778 & 48.5 & 10.9 & 5.69 & 0.01 & 1.95 & $\ldots$ & $\ldots$ & $\mathrm{C}$ \\
\hline 171.170 & 2.589 & 26.2 & 5.3 & 3.18 & -0.84 & 0.78 & $\ldots$ & $\ldots$ & $\mathrm{C}$ \\
\hline 174.712 & 4.478 & 17.5 & 9.7 & 5.26 & -0.03 & 2.38 & $\ldots$ & $\ldots$ & $\mathrm{C}$ \\
\hline 174.761 & 40.549 & 6.4 & 5.5 & 5.29 & -0.11 & 2.36 & $\ldots$ & $\ldots$ & $\mathrm{C}$ \\
\hline 183.954 & 31.860 & -0.6 & 1.4 & 4.13 & -0.17 & 2.26 & $\ldots$ & $\ldots$ & $\mathrm{C}$ \\
\hline 184.145 & 37.391 & -19.8 & -6.4 & 1.78 & -0.87 & 0.83 & $\cdots$ & $\cdots$ & $\mathrm{C}$ \\
\hline 188.053 & 33.928 & -5.2 & -3.9 & 6.0 & -0.88 & 2.48 & $\ldots$ & $\ldots$ & $\mathrm{C}$ \\
\hline 192.636 & 2.276 & -26.9 & -8.5 & 3.05 & -0.03 & 3.50 & $\ldots$ & $\ldots$ & $\mathrm{C}$ \\
\hline 193.189 & 5.284 & -22.9 & 2.2 & 2.96 & -0.78 & 0.63 & $\cdots$ & $\cdots$ & $\mathrm{C}$ \\
\hline 193.593 & 5.772 & -3.1 & 3.7 & 5.72 & -0.24 & 2.25 & $\ldots$ & $\ldots$ & $\mathrm{C}$ \\
\hline 196.183 & 1.542 & -16.3 & -10.4 & 11.19 & -0.14 & 2.29 & $\ldots$ & $\ldots$ & $\mathrm{C}$ \\
\hline 200.482 & 35.177 & 19.6 & 2.8 & 3.33 & -0.59 & 1.92 & $\cdots$ & $\cdots$ & $\mathrm{C}$ \\
\hline 204.568 & 39.475 & 13.1 & 4.5 & 4.09 & -0.52 & 2.46 & $\ldots$ & $\ldots$ & $\mathrm{C}$ \\
\hline 206.403 & 38.387 & 16.7 & 5.3 & 0.54 & -0.65 & 1.85 & $\cdots$ & $\cdots$ & $\mathrm{C}$ \\
\hline 208.298 & 32.095 & -10.3 & 0.3 & 1.89 & -0.69 & 1.56 & $\cdots$ & $\cdots$ & $\mathrm{C}$ \\
\hline 209.863 & 1.998 & 11.4 & 2.6 & 5.86 & 0.05 & 1.33 & $\ldots$ & $\ldots$ & $\mathrm{C}$ \\
\hline
\end{tabular}


Table 1

(Continued)

\begin{tabular}{|c|c|c|c|c|c|c|c|c|c|}
\hline $\begin{array}{l}\text { R.A. } \\
\text { (degrees) }\end{array}$ & $\begin{array}{c}\text { Decl. } \\
\text { (degrees) }\end{array}$ & $\begin{array}{c}\mathrm{RM} \\
\left(\mathrm{rad} \mathrm{m}^{-2}\right)\end{array}$ & $\begin{array}{c}\text { GRM } \\
\left(\mathrm{rad} \mathrm{m}^{-2}\right)\end{array}$ & $\begin{array}{l}\Pi \\
(\%)\end{array}$ & $\alpha$ & $z_{\mathrm{qso}}$ & $z_{\mathrm{abs}}$ & $\log \left[N(\mathrm{H} \mathrm{I}) / \mathrm{cm}^{-2}\right]$ & $\begin{array}{c}\text { Type } \\
(\mathrm{C} / \mathrm{D} / \mathrm{L})\end{array}$ \\
\hline 210.830 & 35.137 & -12.9 & 2.2 & 5.32 & -0.99 & 2.29 & $\ldots$ & $\ldots$ & $\mathrm{C}$ \\
\hline 211.191 & -1.506 & -24.1 & -5.4 & 3.62 & 0.15 & 2.52 & $\ldots$ & $\ldots$ & $\mathrm{C}$ \\
\hline 213.869 & 37.106 & 24.4 & 2.9 & 1.56 & 0.21 & 2.37 & $\ldots$ & $\ldots$ & $\mathrm{C}$ \\
\hline 214.779 & 5.918 & -11.4 & -0.1 & 4.04 & -0.41 & 2.29 & $\ldots$ & $\ldots$ & $\mathrm{C}$ \\
\hline 215.480 & 3.927 & -9.2 & -0.2 & 2.82 & -0.40 & 1.00 & $\ldots$ & $\ldots$ & $\mathrm{C}$ \\
\hline 223.327 & 35.094 & 29.6 & 4.6 & 2.17 & -0.06 & 0.71 & $\ldots$ & $\ldots$ & $\mathrm{C}$ \\
\hline 224.409 & 7.832 & -3.7 & 10.7 & 4.68 & 0.73 & 0.57 & $\ldots$ & $\ldots$ & $\mathrm{C}$ \\
\hline 224.489 & 34.664 & 5.5 & 5.8 & 2.3 & -0.39 & 2.73 & $\ldots$ & $\ldots$ & $\mathrm{C}$ \\
\hline 226.111 & 28.909 & -1.9 & 3.2 & 2.72 & -0.63 & 2.28 & $\ldots$ & $\ldots$ & $\mathrm{C}$ \\
\hline 226.305 & 6.909 & 25.8 & 16.0 & 5.07 & -0.63 & 2.71 & $\ldots$ & $\cdots$ & $\mathrm{C}$ \\
\hline 230.855 & 27.083 & 31.0 & 10.2 & 4.88 & -1.03 & 2.19 & $\ldots$ & $\ldots$ & $\mathrm{C}$ \\
\hline 232.255 & -1.090 & 11.9 & 8.5 & 5.63 & -1.25 & 2.24 & $\ldots$ & $\ldots$ & $\mathrm{C}$ \\
\hline 233.313 & 13.540 & 20.0 & 18.9 & 3.36 & -0.79 & 0.77 & $\ldots$ & $\ldots$ & $\mathrm{C}$ \\
\hline 234.855 & 16.067 & 12.7 & 16.6 & 7.83 & -0.06 & 2.53 & $\ldots$ & $\ldots$ & $\mathrm{C}$ \\
\hline 234.913 & 27.744 & 6.5 & 12.2 & 2.82 & 0.05 & 2.20 & $\ldots$ & $\ldots$ & $\mathrm{C}$ \\
\hline 236.248 & 4.130 & 22.1 & 12.0 & 2.35 & -0.59 & 2.18 & $\ldots$ & $\ldots$ & $\mathrm{C}$ \\
\hline 238.912 & 11.112 & 29.9 & 13.2 & 5.0 & -0.09 & 2.66 & $\ldots$ & $\ldots$ & $\mathrm{C}$ \\
\hline 239.415 & 22.644 & -2.6 & 20.2 & 8.39 & -0.53 & 0.72 & $\ldots$ & $\ldots$ & $\mathrm{C}$ \\
\hline 239.820 & 11.263 & -6.7 & 7.4 & 0.94 & -0.56 & 1.94 & $\ldots$ & $\ldots$ & $\mathrm{C}$ \\
\hline 239.879 & 3.080 & 3.2 & 4.3 & 1.6 & 0.08 & 3.89 & $\ldots$ & $\ldots$ & $\mathrm{C}$ \\
\hline 240.011 & 4.216 & 10.4 & 7.9 & 10.01 & -0.19 & 0.79 & $\ldots$ & $\ldots$ & $\mathrm{C}$ \\
\hline 240.071 & 18.642 & 18.3 & 30.9 & 3.42 & -0.21 & 2.40 & $\ldots$ & $\ldots$ & $\mathrm{C}$ \\
\hline 240.553 & 24.170 & 26.4 & 22.1 & 1.42 & -0.94 & 2.53 & $\ldots$ & $\ldots$ & $\mathrm{C}$ \\
\hline 241.388 & 30.025 & 17.6 & 10.7 & 4.36 & 0.12 & 2.41 & $\ldots$ & $\ldots$ & $\mathrm{C}$ \\
\hline 241.615 & 31.435 & 18.3 & 8.9 & 1.64 & -0.95 & 1.94 & $\ldots$ & $\ldots$ & $\mathrm{C}$ \\
\hline 242.352 & 6.192 & 18.2 & 19.7 & 5.2 & -0.74 & 0.79 & $\ldots$ & $\ldots$ & $\mathrm{C}$ \\
\hline 244.232 & 36.360 & 14.0 & 8.6 & 2.0 & -0.10 & 2.26 & $\ldots$ & $\ldots$ & $\mathrm{C}$ \\
\hline 246.303 & 29.556 & -5.2 & 17.8 & 2.46 & -1.12 & 1.54 & $\ldots$ & $\ldots$ & $\mathrm{C}$ \\
\hline 246.894 & 20.013 & 48.5 & 43.1 & 4.88 & -1.10 & 1.53 & $\ldots$ & $\ldots$ & $\mathrm{C}$ \\
\hline 247.786 & 20.380 & 34.6 & 41.7 & 3.47 & -0.86 & 0.81 & $\ldots$ & $\ldots$ & $\mathrm{C}$ \\
\hline 249.159 & 21.215 & 54.0 & 42.7 & 1.15 & 0.04 & 1.80 & $\ldots$ & $\ldots$ & $\mathrm{C}$ \\
\hline 249.566 & 27.934 & 20.3 & 29.4 & 3.89 & -0.71 & 2.18 & $\ldots$ & $\ldots$ & $\mathrm{C}$ \\
\hline 251.219 & 18.222 & 13.2 & 29.8 & 3.66 & 0.13 & 0.79 & $\cdots$ & $\cdots$ & $\mathrm{C}$ \\
\hline 252.612 & 34.926 & 17.0 & 19.4 & 4.85 & -0.72 & 0.19 & $\ldots$ & $\ldots$ & $\mathrm{C}$ \\
\hline 253.932 & 19.813 & 48.0 & 51.7 & 3.78 & 0.15 & 3.26 & $\ldots$ & $\ldots$ & $\mathrm{C}$ \\
\hline 254.506 & 34.724 & 89.3 & 20.9 & 1.96 & -0.01 & 1.94 & $\ldots$ & $\ldots$ & $\mathrm{C}$ \\
\hline 257.479 & 22.616 & 86.3 & 67.9 & 1.33 & -1.02 & 1.54 & $\ldots$ & $\ldots$ & $\mathrm{C}$ \\
\hline 260.721 & 24.976 & 45.8 & 51.1 & 3.0 & -0.35 & 2.25 & $\ldots$ & $\ldots$ & $\mathrm{C}$ \\
\hline 261.958 & 34.378 & 38.4 & 40.2 & 6.6 & -0.96 & 2.43 & $\ldots$ & $\ldots$ & $\mathrm{C}$ \\
\hline 262.566 & 35.210 & 76.5 & 51.6 & 5.81 & -0.76 & 0.58 & $\ldots$ & $\ldots$ & $\mathrm{C}$ \\
\hline 318.196 & -1.568 & -18.4 & -10.6 & 2.72 & -0.89 & 0.77 & $\ldots$ & $\ldots$ & $\mathrm{C}$ \\
\hline 319.153 & 5.605 & 33.9 & 30.9 & 6.63 & 0.25 & 2.22 & $\ldots$ & $\ldots$ & $\mathrm{C}$ \\
\hline 320.111 & 4.802 & -2.3 & 8.5 & 4.58 & -0.26 & 3.15 & $\ldots$ & $\ldots$ & $\mathrm{C}$ \\
\hline 326.980 & 8.503 & -24.2 & -21.2 & 4.64 & -0.30 & 2.60 & $\ldots$ & $\ldots$ & $\mathrm{C}$ \\
\hline 329.304 & 10.240 & -22.6 & -14.6 & 1.56 & 0.13 & 0.76 & $\cdots$ & $\cdots$ & $\mathrm{C}$ \\
\hline 333.789 & 13.377 & 21.8 & -30.4 & 4.94 & -0.97 & 1.90 & $\cdots$ & $\cdots$ & $\mathrm{C}$ \\
\hline 336.694 & 0.870 & -16.0 & -5.7 & 0.95 & -0.19 & 2.26 & $\ldots$ & $\ldots$ & $\mathrm{C}$ \\
\hline 344.741 & 2.061 & -56.9 & -19.3 & 2.43 & -0.33 & 2.67 & $\ldots$ & $\ldots$ & $\mathrm{C}$ \\
\hline 349.030 & 1.004 & -45.1 & -23.3 & 4.74 & -0.57 & 2.63 & $\ldots$ & $\ldots$ & $\mathrm{C}$ \\
\hline 357.578 & -0.116 & 20.2 & -5.0 & 1.86 & -0.86 & 1.37 & $\ldots$ & $\ldots$ & $\mathrm{C}$ \\
\hline 359.620 & 4.507 & -52.9 & -6.5 & 1.37 & 0.24 & 2.30 & $\ldots$ & $\ldots$ & $\mathrm{C}$ \\
\hline 20.132 & -27.024 & 18.6 & 12.3 & 4.03 & -0.10 & 0.56 & 0.56 & 20.3 & $\mathrm{D}$ \\
\hline 30.944 & 11.579 & -3.8 & -11.5 & 1.54 & 0.01 & 3.61 & 3.39 & 21.3 & $\mathrm{D}$ \\
\hline 33.654 & 6.548 & 3.4 & -0.9 & 3.86 & -0.37 & 2.31 & 2.11 & 20.8 & $\mathrm{D}$ \\
\hline 39.662 & 16.616 & 42.0 & -0.7 & 2.02 & 0.00 & 0.94 & 0.52 & 21.6 & $\mathrm{D}$ \\
\hline 54.754 & -1.555 & 26.9 & 22.7 & 4.71 & -0.10 & 3.20 & 3.06 & 21.2 & $\mathrm{D}$ \\
\hline 57.491 & -21.047 & 26.2 & 33.0 & 1.44 & 0.42 & 2.94 & 1.95 & 20.3 & $\mathrm{D}$ \\
\hline 61.891 & -33.063 & 4.2 & 8.2 & 3.09 & -0.03 & 2.57 & 2.57 & 20.6 & $\mathrm{D}$ \\
\hline 82.533 & -25.058 & 32.2 & 24.8 & 0.68 & -0.41 & 2.78 & 2.14 & 20.6 & $\mathrm{D}$ \\
\hline 127.717 & 24.183 & 12.0 & 15.4 & 8.75 & 0.00 & 0.94 & 0.52 & 20.3 & $\mathrm{D}$ \\
\hline 138.965 & 0.120 & 61.3 & -0.4 & 4.78 & -0.50 & 3.07 & 2.77 & 20.3 & $\mathrm{D}$ \\
\hline 140.149 & 0.392 & 1.1 & -3.4 & 4.66 & -0.40 & 2.48 & 2.04 & 20.6 & $\mathrm{D}$ \\
\hline 148.737 & 17.725 & -5.5 & 2.5 & 4.42 & -0.24 & 1.48 & 0.24 & 21.3 & $\mathrm{D}$ \\
\hline
\end{tabular}


Table 1

(Continued)

\begin{tabular}{|c|c|c|c|c|c|c|c|c|c|}
\hline $\begin{array}{l}\text { R.A. } \\
\text { (degrees) }\end{array}$ & $\begin{array}{c}\text { Decl. } \\
\text { (degrees) }\end{array}$ & $\begin{array}{c}\mathrm{RM} \\
\left(\operatorname{rad~m}^{-2}\right)\end{array}$ & $\begin{array}{c}\text { GRM } \\
\left(\operatorname{rad~m}^{-2}\right)\end{array}$ & $\begin{array}{c}\Pi \\
(\%)\end{array}$ & $\alpha$ & $z_{\mathrm{qso}}$ & $z_{\mathrm{abs}}$ & $\log \left[N(\mathrm{H} \mathrm{I}) / \mathrm{cm}^{-2}\right]$ & $\begin{array}{c}\text { Type } \\
(\mathrm{C} / \mathrm{D} / \mathrm{L})\end{array}$ \\
\hline 172.529 & -14.824 & 34.0 & -14.0 & 4.44 & -0.05 & 1.19 & 0.31 & 21.7 & D \\
\hline 187.732 & -11.653 & -36.2 & -11.8 & 0.98 & -0.57 & 3.53 & 2.19 & 20.6 & D \\
\hline 202.785 & 30.509 & 8.8 & 1.3 & 9.16 & -0.57 & 0.85 & 0.69 & 21.3 & $\mathrm{D}$ \\
\hline 232.466 & 19.076 & -29.0 & 14.7 & 2.55 & -0.56 & 2.36 & 2.22 & 20.7 & $\mathrm{D}$ \\
\hline 239.654 & 24.637 & 26.8 & 20.5 & 15.67 & $\ldots$ & 3.35 & 2.20 & 20.3 & D \\
\hline 247.939 & 11.934 & 39.9 & 34.3 & 2.37 & -0.52 & 1.79 & 0.53 & 20.7 & D \\
\hline 332.734 & 1.986 & -6.7 & -14.3 & 5.3 & -0.17 & 2.59 & 2.39 & 20.5 & D \\
\hline 20.616 & -4.358 & 27.7 & 0.1 & 2.55 & -0.67 & 1.95 & 0.66 & 18.8 & $\mathrm{~L}$ \\
\hline 34.454 & 1.747 & -17.5 & -9.6 & 1.41 & 0.43 & 1.72 & 1.34 & 19.9 & $\mathrm{~L}$ \\
\hline 65.816 & -1.342 & -25.5 & -22.9 & 1.67 & 0.52 & 0.92 & 0.63 & 18.5 & $\mathrm{~L}$ \\
\hline 118.264 & 42.525 & 147.0 & 18.1 & 1.25 & -0.14 & 3.59 & 3.22 & 18.6 & $\mathrm{~L}$ \\
\hline 129.981 & 2.863 & -3.0 & 32.5 & 5.07 & -0.24 & 3.68 & 3.57 & 17.4 & $\mathrm{~L}$ \\
\hline 131.447 & 13.483 & 10.6 & 13.8 & 4.49 & -0.61 & 1.88 & 0.61 & 19.6 & $\mathrm{~L}$ \\
\hline 133.238 & 24.517 & 13.1 & 21.5 & 1.8 & -0.73 & 3.62 & 3.34 & 17.0 & $\mathrm{~L}$ \\
\hline 159.414 & 4.400 & 76.8 & 11.5 & 2.53 & 0.12 & 2.32 & 2.15 & 20.2 & $\mathrm{~L}$ \\
\hline 160.321 & 6.171 & 25.0 & 15.2 & 2.17 & 0.09 & 1.26 & 0.44 & 18.3 & $\mathrm{~L}$ \\
\hline 160.686 & 12.059 & 25.5 & 14.7 & 6.88 & -0.51 & 1.03 & 0.66 & 18.4 & $\mathrm{~L}$ \\
\hline 163.136 & 61.422 & -1.6 & -0.8 & 3.9 & -0.80 & 0.42 & 0.23 & 18.0 & $\mathrm{~L}$ \\
\hline 177.872 & 38.431 & 8.4 & 1.8 & 4.02 & -0.75 & 1.30 & 0.55 & 18.0 & $\mathrm{~L}$ \\
\hline 187.153 & 10.312 & 2.6 & 2.5 & 3.24 & -0.80 & 2.31 & 0.94 & 19.4 & $\mathrm{~L}$ \\
\hline 190.541 & 37.335 & -14.6 & -7.0 & 2.46 & -0.13 & 3.82 & 3.01 & 20.0 & $\mathrm{~L}$ \\
\hline 190.541 & 37.335 & -14.6 & -7.0 & 2.46 & -0.13 & 3.84 & 3.41 & 17.6 & $\mathrm{~L}$ \\
\hline 191.045 & 17.351 & -1.0 & 1.4 & 1.38 & -0.35 & 1.28 & 0.55 & 18.9 & $\mathrm{~L}$ \\
\hline 201.373 & 65.254 & 40.2 & 33.3 & 3.31 & -0.88 & 1.62 & 1.52 & 18.6 & $\mathrm{~L}$ \\
\hline 208.359 & 57.431 & 55.7 & 15.0 & 2.68 & -0.66 & 3.47 & 3.46 & 17.8 & $\mathrm{~L}$ \\
\hline 208.529 & -2.101 & -7.5 & -2.2 & 0.83 & -0.01 & 3.72 & 3.45 & 18.2 & $\mathrm{~L}$ \\
\hline 209.268 & 19.319 & 28.1 & 7.9 & 6.14 & 0.02 & 0.72 & 0.46 & 18.5 & $\mathrm{~L}$ \\
\hline 218.891 & 54.600 & 14.6 & 12.7 & 4.26 & -0.23 & 3.81 & 3.81 & 17.0 & $\mathrm{~L}$ \\
\hline 224.863 & 32.900 & 28.1 & 7.7 & 4.7 & -0.91 & 3.33 & 2.23 & 20.1 & $\mathrm{~L}$ \\
\hline 251.553 & 15.617 & 47.5 & 32.2 & 6.1 & -0.23 & 2.86 & 2.77 & 20.3 & $\mathrm{~L}$ \\
\hline 322.897 & -12.118 & 13.0 & 9.9 & 1.93 & 0.14 & 0.50 & 0.43 & 19.2 & $\mathrm{~L}$ \\
\hline 323.550 & -4.319 & -7.4 & -7.2 & 4.83 & -0.49 & 4.33 & 3.27 & 20.0 & $\mathrm{~L}$ \\
\hline 324.755 & 14.393 & -40.6 & -34.0 & 0.87 & 0.10 & 2.43 & 2.13 & 19.8 & $\mathrm{~L}$ \\
\hline 336.447 & -4.951 & -22.9 & -11.8 & 4.81 & -0.09 & 1.40 & 0.85 & 18.5 & $\mathrm{~L}$ \\
\hline
\end{tabular}

Note. All of the source names, uncertainties, and more extensive source parameters can be found in Taylor et al. (2009), Farnes et al. (2014a), and Oppermann et al. (2015). The control, DLA, and LLS samples are denoted "C," "D," and "L," respectively. Blanks entries have no listed value.

To answer our scientific question, we want to know: what is the probability that the RMs of the DLA (or LLS) sample are smaller than the RMs of the control sample, given our data? Or equivalently, for the DLAs,

$$
p(\mathrm{E}[\Pi(\mathrm{DLA})]<\mathrm{E}[\Pi(\text { control })] \mid \mathrm{D}) .
$$

We evaluate Equation (3), for the controls, DLAs, and LLSs, through numerical integration, as described in Section 3.2. The obtained probabilities are given in Table 4 .

\subsection{Robustness Checks}

Robustness checks are important tests of the validity of our results. The nature of robustness checks are covered in Gelman et al. (2004). The checks themselves do not modify our results, unless the check highlights some factor that drastically changes our conclusions.

\subsubsection{Prior Selection}

First, we wish to ensure that our results are not unduly sensitive to the choice of prior, which is explained in detail in the Appendix. We expect no significant change, particularly as we have only used weakly informative priors throughout our analysis. We therefore repeat our analysis with an uninformative prior, this time critically setting $\kappa_{0}=0.0$ (equivalent to no prior measurements) to obtain a uniform distribution. Our results remain essentially unchanged, and the redetermined probabilities are given in Table 5. Using the uninformative prior, we find a $30.8 \%$ probability that the DLAs have lower $|\mathrm{RM}|$ than the controls, or equivalently a $69.2 \%$ probability that the controls have higher $|\mathrm{RM}|$ than the DLAs. Note that this does not in any way modify our results, as this is a robustness check and shows a change of only $1.3 \%$. In addition, the DLAs having lower $|\mathrm{RM}|$ would be unphysical. This probability did not hold when we incorporated some physical information into the weakly informative prior that we used for our actual analysis.

\subsubsection{Non-intervenors}

As we have a sample of limited size, we cannot substantially subdivide or split the sample without losing meaningful statistical power. This limits our ability to explore other connections with these data, such as the evolution of magnetic 
Table 2

A Statistical Overview for All of the Polarized QSOs Used in our Analysis

\begin{tabular}{|c|c|c|c|c|c|c|c|c|}
\hline & $\begin{array}{c}\mathrm{RM} \\
\left(\mathrm{rad} \mathrm{m}^{-2}\right)\end{array}$ & $\begin{array}{c}\mathrm{GRM} \\
\left(\mathrm{rad} \mathrm{m}^{-2}\right)\end{array}$ & $\begin{array}{c}\Pi \\
(\%)\end{array}$ & $\alpha$ & $z_{\mathrm{qso}}$ & $z_{\mathrm{abs}}$ & $\log \left[N(\mathrm{H} \mathrm{I}) / \mathrm{cm}^{-2}\right]$ & $\begin{array}{c}\text { Type } \\
(\mathrm{C} / \mathrm{D} / \mathrm{L})\end{array}$ \\
\hline Mean & 8.9 & 10.3 & 4.20 & -0.44 & 1.97 & $\ldots$ & $\ldots$ & $\mathrm{C}$ \\
\hline Median & 10.1 & 8.6 & 3.97 & -0.47 & 2.23 & $\ldots$ & $\ldots$ & $\mathrm{C}$ \\
\hline Std. Dev. & 26.5 & 16.7 & 2.53 & 0.41 & 0.80 & $\ldots$ & $\ldots$ & $\mathrm{C}$ \\
\hline Mean & 13.5 & 7.0 & 4.44 & -0.23 & 2.24 & 1.71 & 20.79 & D \\
\hline Median & 12.0 & 2.5 & 4.03 & -0.20 & 2.48 & 2.11 & 20.63 & $\mathrm{D}$ \\
\hline Std. Dev. & 23.9 & 15.0 & 3.46 & 0.27 & 0.96 & 1.00 & 0.45 & $\mathrm{D}$ \\
\hline Mean & 15.1 & 5.5 & 3.25 & -0.29 & 2.34 & 1.84 & 18.76 & $\mathrm{~L}$ \\
\hline Median & 10.6 & 7.7 & 2.68 & -0.23 & 2.31 & 1.52 & 18.56 & $\mathrm{~L}$ \\
\hline Std. Dev. & 36.7 & 15.7 & 1.68 & 0.40 & 1.20 & 1.26 & 0.97 & $\mathrm{~L}$ \\
\hline
\end{tabular}

fields with column density, redshift, or spectral index. However, to ensure that our results are correct, we can perform robustness checks on our data in which we exclude a small fraction of sources at a time to ensure a similar distribution of source parameters between all samples, and hence attempt to control for and minimize any selection effects.

There are two components to this particular experiment. One is in measuring magnetic fields associated with DLAs/LLSs (more generally), and the other is in measuring magnetic fields in only the intervening DLAs/LLSs (unassociated with the background QSO). Both are unanswered scientific questions. While measuring the magnetic environment of DLAs and LLSs is of interest, the primary motivation is in observing the intervening systems that are unrelated to the background quasar itself. If the QSO and the intervenor are in close proximity to one another, then there is a possibility that their magnetic fields are not independent. This can be ruled out by removing systems from our sample that have nearby QSOs and intervenors, and confirming whether nothing changes. One robustness check therefore requires ensuring that our results remain unchanged when only selecting the intervening DLA and LLS lines of sight. We therefore repeat our analysis, this time excluding the few sources with $\Delta z=z_{\mathrm{qso}}-z_{\mathrm{abs}} \leqslant 0.04$. Our results remain essentially unchanged, and the redetermined probabilities are given in Table 5. Given our data, there is no evidence for strong magnetic fields present in DLAs and LLSs associated with the background quasars themselves. Moreover, one of the sources in our sample-located at (R.A., decl. $)=\left(190^{\circ} .541,37^{\circ} .335\right)$ - has two LLSs located along the line of sight. As a robustness check, this source was excluded from our analysis, and our results again remain unchanged.

\subsubsection{Spectral Distribution}

Following a rationale equivalent to that in Section 3.4.2, we also wish to ensure that our samples (the control, DLA, and LLS samples, respectively) all have a similar distribution of spectral index. From the distribution of spectral index shown in Figure 3, it could be argued that the control sample shows a slight enhancement of steep-spectrum sources with $\alpha \leqslant-1.0$ in contrast to the DLA and LLS samples.

We therefore repeat our analysis, this time excluding the few sources with $\alpha \leqslant-1.0$. Our results remain essentially unchanged, and the redetermined probabilities are given in Table 5 .

\subsubsection{Source Size}

Following a rationale equivalent to that in Section 3.4.2, we also wish to ensure that our samples (the control, DLA, and LLS samples, respectively) all have a similar distribution of source size. From the distribution of effective area shown in Figure 4, it could be argued that the control sample shows a slight enhancement of more extended sources with $A \geqslant 350 \operatorname{arcsec}^{2}$ in contrast to the DLA and LLS samples.

We therefore repeat our analysis, this time excluding the few sources with $A \geqslant 350 \operatorname{arcsec}^{2}$. Our results remain essentially unchanged, and the redetermined probabilities are given in Table 5. We also repeat our analysis when only including the sources with a major-axis limit $\leqslant 25$ arcsec. This selects the same sample as our source area cut on the data, and our results therefore remain unchanged. Future observations with higher angular resolution data will be able to further test source size effects; however, this clearly cannot explain the strength of the observed correlations in our data.

\subsubsection{Redshift Distribution}

Following a rationale equivalent to that in Section 3.4.2, we also wish to ensure that our samples (the control, DLA, and LLS samples, respectively) all have a similar distribution of redshifts of the background QSOs, otherwise it could be argued that any difference between samples originates from differences in the selected QSOs. From the distribution of redshifts shown in Figure 6, it could be argued that the DLA and LLS samples show an enhancement of sources with $z_{\text {qso }} \geqslant 3.5$ in contrast to the control sample.

We therefore repeat our analysis, this time excluding the few sources with $z_{\mathrm{qso}} \geqslant 3.5$. The redetermined probabilities are given in Table 5. The majority of our results remain essentially unchanged, however, with two notable exceptions. First, the probability that the $|\mathrm{RM}|$ of the DLAs is higher than the controls has reduced to $17.4 \%$, or equivalently an $82.6 \%$ probability that the controls have higher $|\mathrm{RM}|$ than the DLAs. Note that this does not in any way modify our results, as the DLAs having lower $|\mathrm{RM}|$ would be unphysical. Second, the probability that the $|\mathrm{RM}|$ of the LLSs is greater than the controls has reduced to $71.5 \%$. This could be a mild indication of an enhanced $|\mathrm{RM}|$ associated with the LLSs; however, it is a significant drop in probability of almost $30 \%$ from when we do not control for the redshift distributions. We interpret this to indicate only a mild detection of enhanced $|\mathrm{RM}|$ associated with the LLSs, and that we are most likely currently sensitive to the high-redshift distribution of QSOs. We therefore consider 

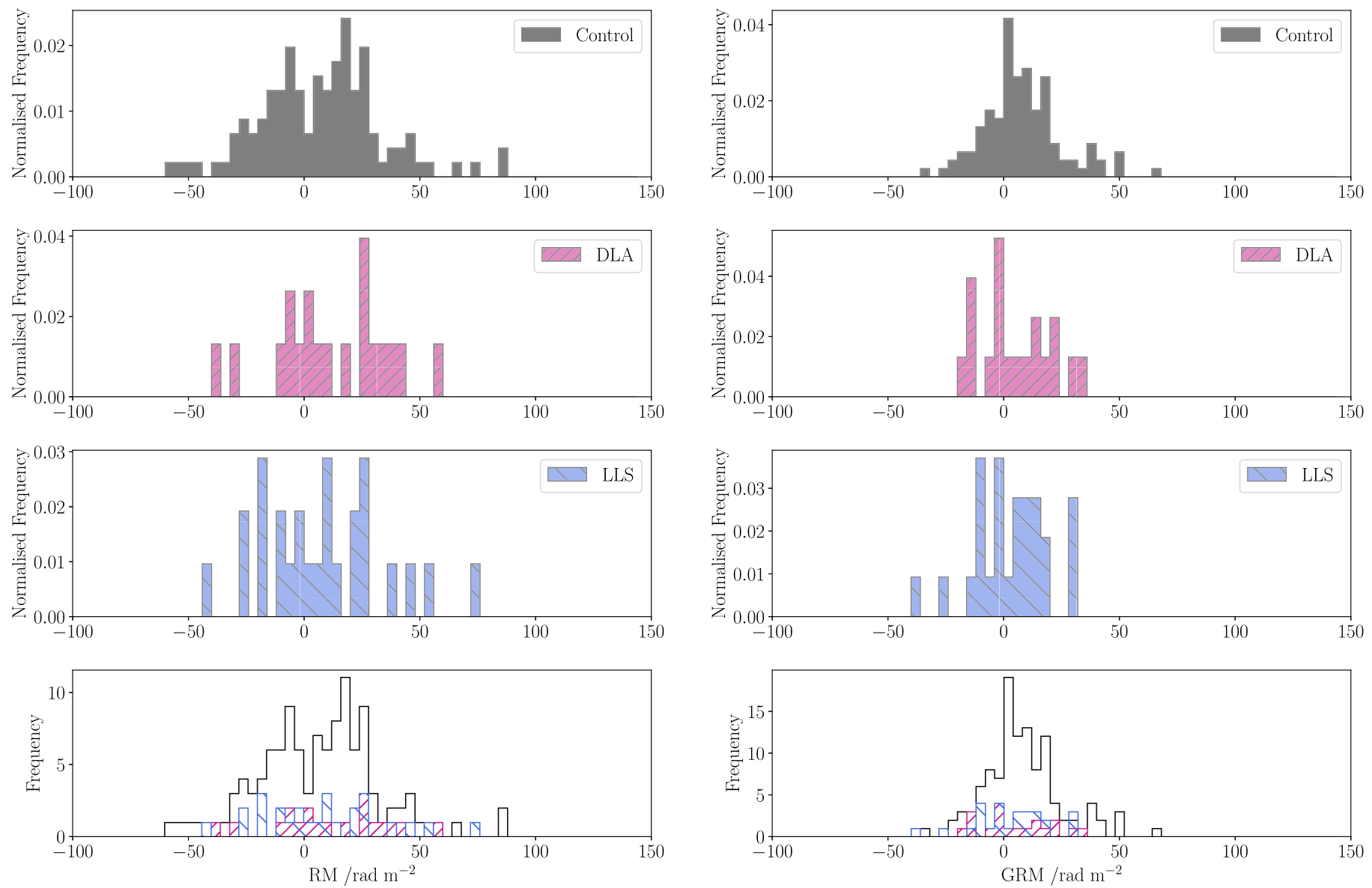

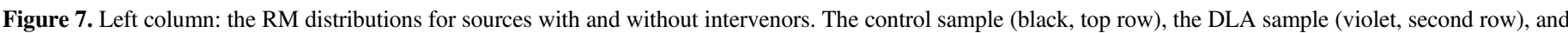

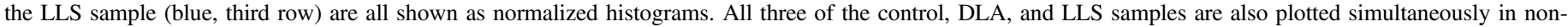

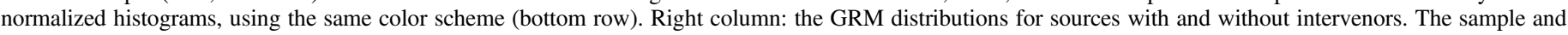
color scheme is the same as in the left column.

$71.5 \%$ to be the more conservative estimate. For the $|\mathrm{RM}|$ measurements of the LLSs, we are therefore unable to fully control for the redshift distribution of the data. Removing the upper range of high- $z$ sources works as a rough approximation, although it is a fairly arbitrary approach. Future tests of the mutual consistency between data sets will be useful for similar experiments (Karpenka et al. 2015).

\subsubsection{Sky Distribution}

The sources are distributed across the sky. As the magnetized Galactic foreground varies as a function of sky position, this makes the results sensitive to the sky distribution of sources. One possibility to explain the apparent difference between the $|\mathrm{RM}|$ of LLSs and a control sample is that it is the consequence of contributions to the Faraday rotation from the Galactic foreground. Similarly, one could argue that the RM distribution is also responsible for the different polarized fractions. If the Galactic Faraday screen responsible for higher $|R M|$ is also responsible for increased depolarization, then one would expect a lower polarized fraction for the LLSs.

Currently, the Galactic foreground cannot be reliably subtracted to obtain an RRM (see Section 3.2). Regardless, we expect the effect of the Galactic foreground to be low, as we have removed sources at low Galactic latitudes from our sample (see Section 2) and we would expect sources with high $|R M|$ to be preferentially located in the Galactic plane (Taylor et al. 2009). For the foreground to be influencing our main results, our sample would have to be anisotropically distributed on the sky such that there was a difference in the $|R M|$ of sources with and without absorbing systems. Therefore, if the Galactic foreground was causing our result, we could expect a different estimation of GRM between these different samples.

We have found a $38.2 \%$ probability that the GRM is different in the control and DLA samples. Similarly, we have found a $39.8 \%$ probability that the GRM is different in the control and LLS samples. There is therefore no indication that a varying Galactic foreground is influencing our results. The statistical differences in the RMs, with no associated difference in the GRMs, demonstrates that the LLSs cannot have significantly different sky distributions (relative to the Faraday structure of the Milky Way). We have therefore ruled out our results being affected by the sky distribution of sources.

\section{Discussion}

\subsection{Ionized Gas in DLAs and LLSS}

We are interested in proxies for characteristics of the magnetic field - in particular, measurements of Faraday rotation, via the RM, and of magnetic field disorder or free electron variations via the polarized fraction (which is itself a proxy for the depolarization). In particular, measuring an RM fundamentally requires the presence of ionized or partially ionized gas that is permeated by magnetic fields. 

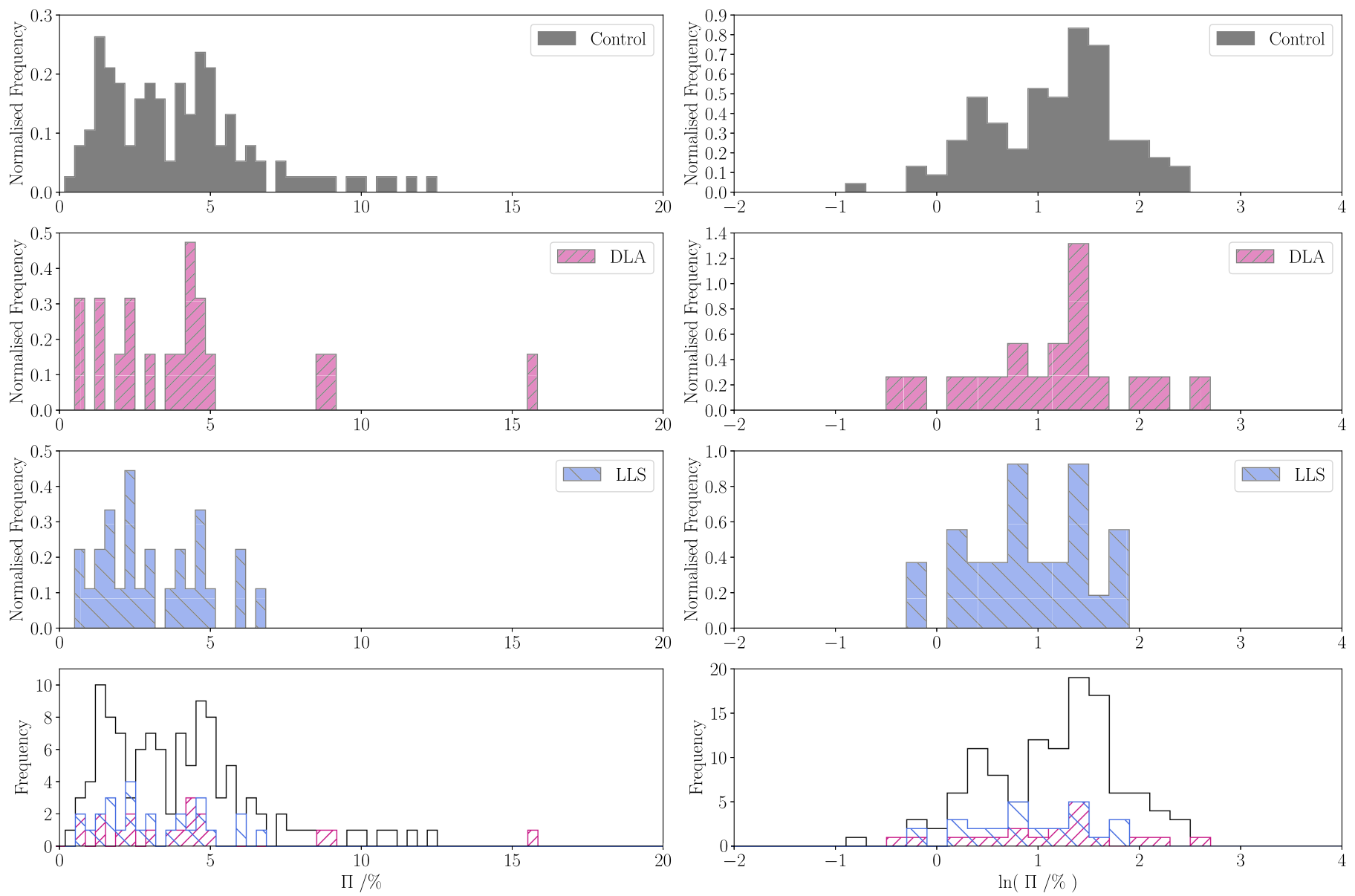

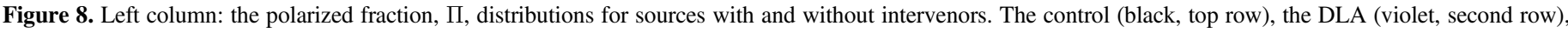

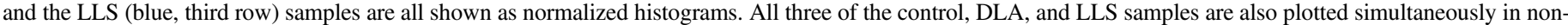

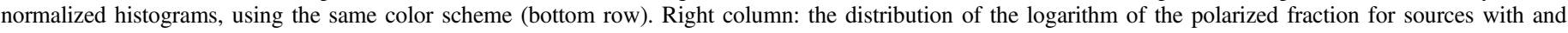
without intervenors. The samples and color scheme are the same as in the left column.

Understanding how this ionization affects our measurements requires consideration of the physical nature of DLAs and LLSs as detailed in Section 1.1. Are the DLAs and LLSs physically different from one another, or are we imposing arbitrary distinctions due to the column density criterion? There is a critical and fundamental distinction between the systems: the hydrogen gas is mostly neutral in DLAs, while the gas is ionized in other absorption systems such as LLSs.

The neutrality of the gas in DLAs is crucial to their important role as neutral gas reservoirs for star formation in the highredshift universe (Wolfe et al. 2005). The birthplace of starsmolecular clouds - are typically preceded and formed from cold neutral clouds, from which stars are able to form. However, stars are unlikely to form from warm ionized gas. The DLAs therefore enable a view into the interaction between neutral gas and new star formation, and are potentially the only example of an ISM at high redshifts. DLAs also remain predominantly neutral due to "self-shielding," which prevents ionization of the gas. While ultraviolet radiation ionizes the gas, at these extreme high column densities the ionized material rapidly recombines and becomes neutral again (Wolfe \& Prochaska 2000).

At lower column densities, it is unclear where the transition from predominantly neutral to predominantly ionized gas occurs, except that it is below the defining column density of DLAs (log $N(\mathrm{H}$ I $) \geqslant 20.3$; Prochaska \& Wolfe 1997; Haehnelt et al. 1998;
Maller et al. 2001). In both the LLSs $(17<\log N(\mathrm{H} \mathrm{I})<20.3)$ and the Ly $\alpha$ forest $(\log N(\mathrm{HI}) \leqslant 17)$, the neutral gas is a minor or non-existent phase, and so these lower column density systems primarily trace ionized gas. However, as the H I column density increases from the threshold of an LLS up to a DLA, the systems go from mostly ionized to mostly neutral due to the selfshielding (Erkal 2015). As such, the majority of the hydrogen gas in DLAs is typically considered to be completely neutral (with a negligible ionization fraction) in the H I phase (Meiring et al. 2009).

Nevertheless, there is actually a small amount of ionized gas in DLAs, as evidenced by the highly ionized metal absorption lines seen in many DLAs. For example, Lehner et al. (2008) found that the ionization fraction $\mathrm{H} \mathrm{II} / \mathrm{H}$ is about $12 \%-20 \%$ in DLAs. Radio observations of ionized gas in the Galactic disk of the Milky Way have shown that the mass ratio $^{6}$ of $\mathrm{H}$ II to $\mathrm{H} \mathrm{I}$ gases is $\approx 0.01$ (Osterbrock 1989; Okoshi \& Nagashima 2005). Meanwhile, Prochaska et al. (2002) and Fox et al. (2011) reported a hydrogen ionization fraction in DLAs of $\bar{x}(\mathrm{HI})=$ $10 \%-50 \%$. Furthermore, Prochaska \& Wolfe (1996) calculated the ionization fraction in DLAs by taking radiation transfer into account and assuming an ultraviolet background intensity corresponding to that at $z \sim 2-3$. They found the ionization fraction $\bar{x}\left(n_{e} / n\right)=4 \%$.

\footnotetext{
This is not the filling factor or volume ratio, but the mass ratio.
} 

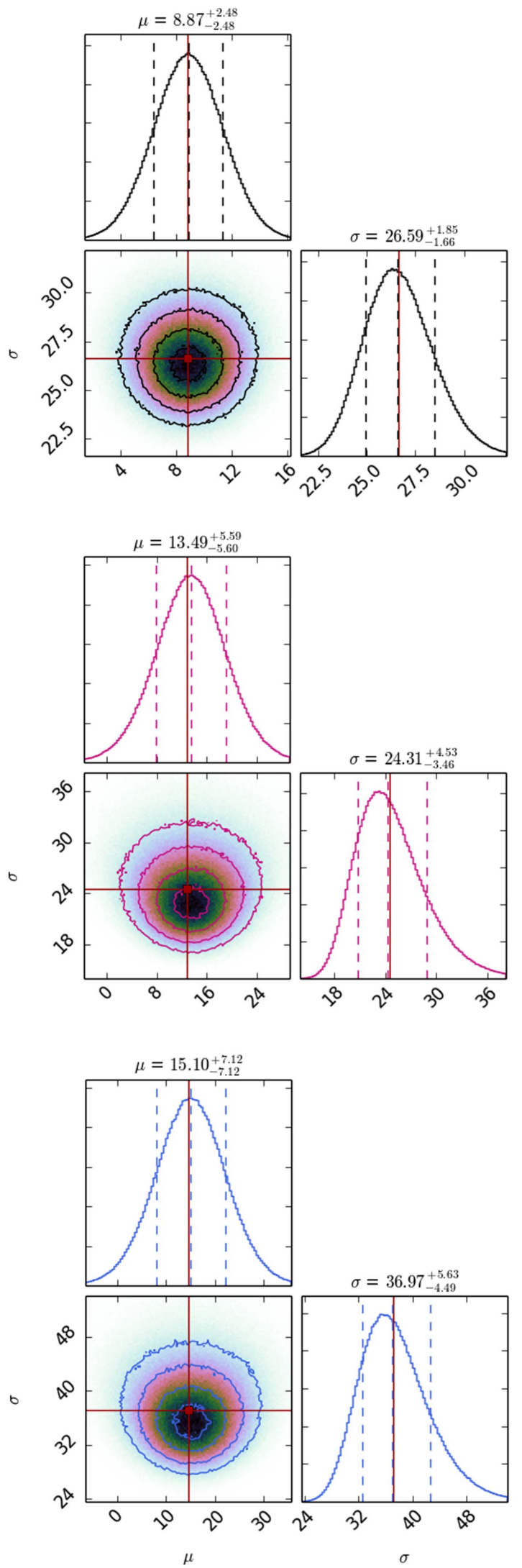
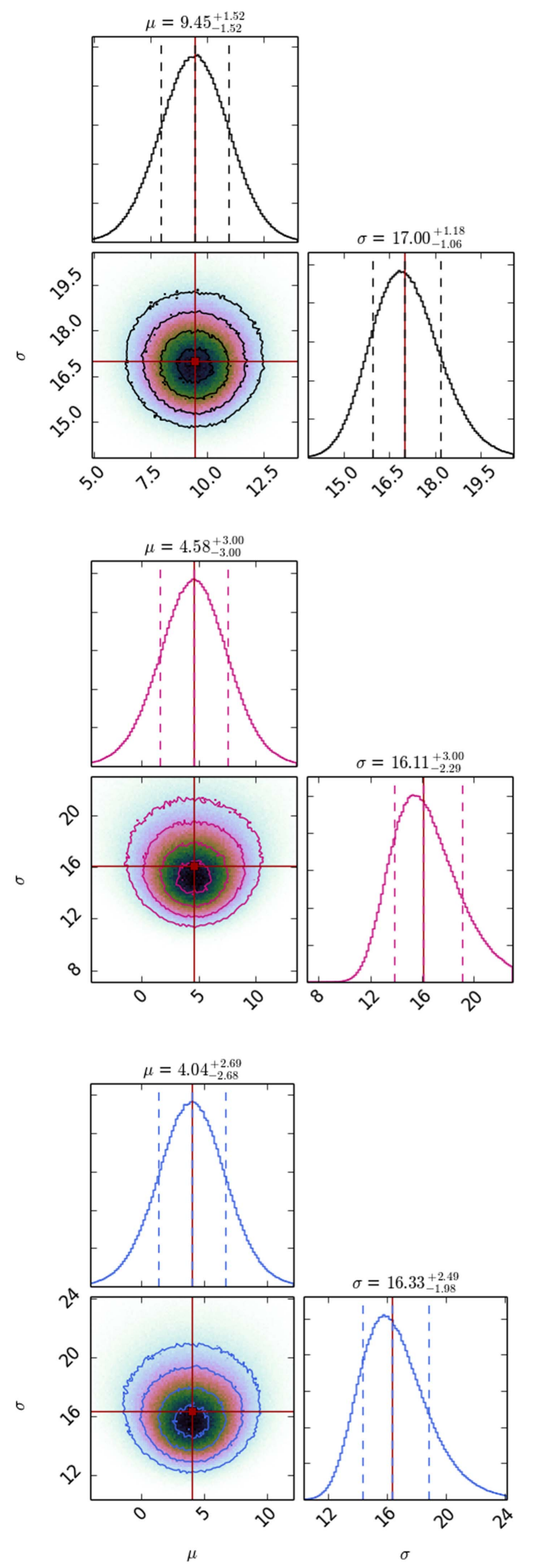

Figure 9. Parameter estimation of the RMs (left column) and the GRMs (right column). The estimates from the control (black, top row), the DLA (violet, middle row), and the LLS sample (blue, bottom row) are all shown. The parameter estimates for $\mu$ and $\sigma$ are stated in units of rad $\mathrm{m}^{-2}$. The estimated parameters are shown by the solid line, indicating the median, and the dashed lines, indicating the $68 \%$ credible interval. The sample density is shown on a pseudocolor scale using the cubehelix color scheme (Green 2011), and contour levels are shown at $11.7 \%, 39.3 \%, 67.5 \%$, and $86.5 \%$. 
Table 3

Calculated $p\left(\mathrm{RM}_{A}>\mathrm{RM}_{B}\right)$ and $p\left(\mathrm{GRM}_{A}>\mathrm{GRM}_{B}\right)$ for the Control, DLA, and LLS Samples

\begin{tabular}{lc}
\hline \hline Probability & $\%$ \\
\hline$\sigma_{\mathrm{RM}}($ DLA $)>\sigma_{\mathrm{RM}}($ control $)$ & 32.1 \\
$\sigma_{\mathrm{RM}}($ LLS $)>\sigma_{\mathrm{RM}}($ control $)$ & 99.0 \\
$\sigma_{\mathrm{RM}}($ LLS $)>\sigma_{\mathrm{RM}}($ DLA $)$ & 97.1 \\
$\sigma_{\mathrm{GRM}}($ DLA $)>\sigma_{\mathrm{GRM}}($ control $)$ & 38.2 \\
$\sigma_{\mathrm{GRM}}($ LLS $)>\sigma_{\mathrm{GRM}}($ control $)$ & 39.8 \\
$\sigma_{\mathrm{GRM}}($ LLS $)>\sigma_{\mathrm{GRM}}($ DLA $)$ & 52.6 \\
\hline
\end{tabular}

In contrast, the LLSs definitively trace ionized gas. In one system, an ionization fraction of $97 \% \pm 2 \%$ was reported (Prochaska 1999). Meanwhile, the LLSs with highest column densities $(19<\log N(\mathrm{HI})<20.3)$, sometimes referred to as the Super Lyman Limit Systems (SLLS), have been found to have mean ionization fractions of $90 \%$ (Péroux et al. 2007; Prochaska \& Tumlinson 2009). Furthermore, a study of 157 systems confirmed that the majority of LLSs are highly ionized and also showed that there is an increasing ionization state of the gas with decreasing column density (Prochaska et al. 2015).

\subsection{Coherent Magnetic Fields}

We can use our measurements of the RM and GRM associated with the absorption line systems, relative to the control sample, in order to place constraints on the coherent magnetic field strength in the DLAs and the LLSs. ${ }^{7}$ We assume throughout that any change in the data resembles a change in the overall population, rather than the emergence of a significant number of physical outliers in one of the samples. Such a scenario could occur if, for example, there were two populations of sources in one of our samples, perhaps one population having undergone significant dynamo activity and the other population having not. Such an alternative could only be addressed with much better statistics.

Following Kronberg \& Perry (1982), for an RRM excess measured in the observing frame, $\triangle \mathrm{RRM}$, which arises in the rest frame at redshift $z$,

$$
\Delta \mathrm{RRM}=\beta(1+z)^{-2} N_{e}\left\langle B_{\|}\right\rangle,
$$

where $N_{e}$ is the electron column density in $\mathrm{cm}^{-2}, \beta=2.63 \times$ $10^{-19} \mathrm{rad} \mathrm{m}^{-2} \mathrm{~cm}^{-2} \mu \mathrm{G}^{-1}$, and $\left\langle B_{\|}\right\rangle$for any field reversal pattern is

$$
\left\langle B_{\|}\right\rangle=\frac{\int n_{e} B_{\|} d l}{\int n_{e} d l},
$$

where $n_{e}$ is the number density of free electrons in $\mathrm{cm}^{-3}, B_{\|}$is the component of the magnetic field parallel to the line of sight in $\mu \mathrm{G}$, and $d l$ is a finite element of the path length in parsec. The integrals are defined from us to the background source. The line-of-sight component of an intervenor's magnetic field

\footnotetext{
7 Note that it is not possible to use the total intensity radio continuum emission to calculate an equipartition magnetic field in the DLAs and LLSs, as the emission from the absorbers is several orders of magnitude fainter than that from the bright background QSO.
}
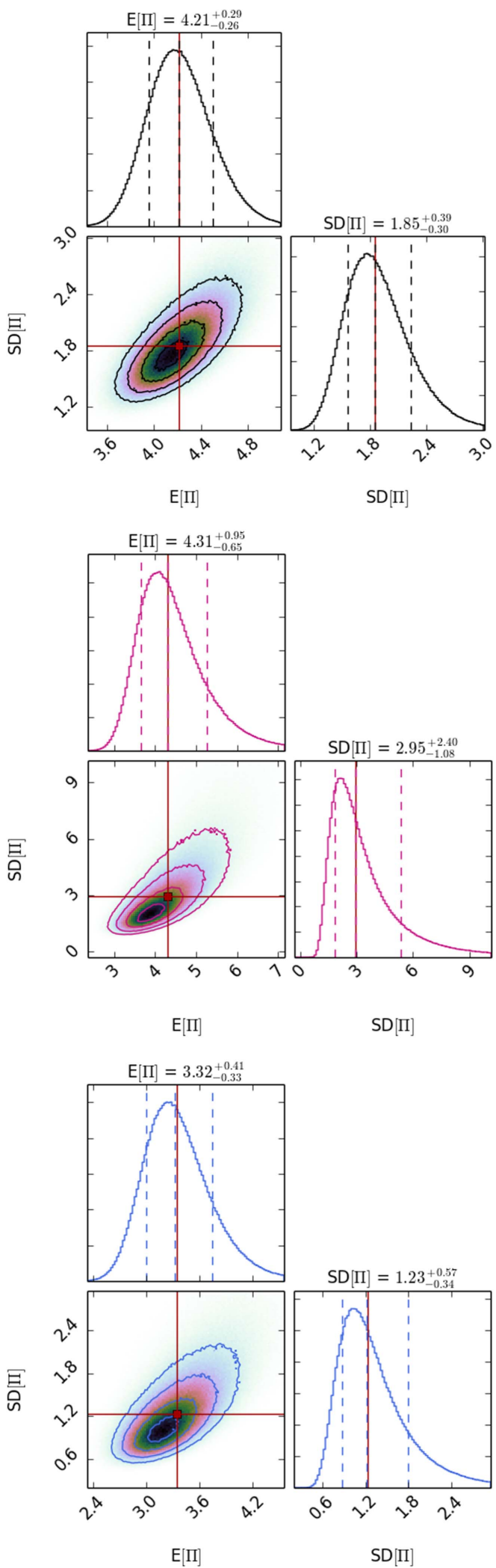

Figure 10. Parameter estimation of the polarized fractions, $\Pi$. The estimates from the control (black, top row), the DLA (violet, middle row), and the LLS sample (blue, bottom row) are all shown. The parameter estimates for $\mathrm{E}[\Pi]$ and $\mathrm{SD}[\Pi]$ are stated in units of percent. The estimated parameters are shown by the solid line, indicating the median, and the dashed lines, indicating the $68 \%$ credible interval. The sample density is shown on a pseudocolor scale, and contour levels are shown at $11.7 \%, 39.3 \%, 67.5 \%$, and $86.5 \%$. 
Table 4

Calculated $p\left(\mathrm{E}\left[\Pi_{A}\right]<\mathrm{E}\left[\Pi_{B}\right]\right)$ for the Control, DLA, and LLS Samples

\begin{tabular}{lc}
\hline \hline Probability & $\%$ \\
\hline $\mathrm{E}[\Pi(\mathrm{DLA})]<\mathrm{E}[\Pi($ control) $]$ & 45.2 \\
$\mathrm{E}[\Pi(\mathrm{LLS})]<\mathrm{E}[\Pi($ control) $]$ & 95.5 \\
$\mathrm{E}[\Pi(\mathrm{LLS})]<\mathrm{E}[\Pi($ DLA) $]$ & 90.0 \\
\hline
\end{tabular}

is therefore given by

$$
\left\langle B_{\|}\right\rangle=\frac{\Delta \operatorname{RRM}(1+z)^{2}}{\beta N_{e}} .
$$

The electron column density is related to the neutral hydrogen column density, $N(\mathrm{H} \mathrm{I})$, by

$$
N_{e} \approx \frac{\bar{x}}{(1-\bar{x})} N(\mathrm{H} \mathrm{I}) \text {, }
$$

where $\bar{x}$ is the hydrogen ionization fraction. Consequently, we can write

$$
\left\langle B_{\|}\right\rangle=\frac{(1-\bar{x}) \Delta \mathrm{RRM}(1+z)^{2}}{\beta \bar{x} N(\mathrm{H} \mathrm{I})} .
$$

In our calculations, we use a small ionization fraction of $5 \%$ in DLAs and an ionization fraction of $95 \%$ in LLSs. These estimates are justified in Section 4.1. For DLAs, the low ionization state limits the expected RRM excess; however, the very high column density ensures that even a small ionization fraction can result in a large electron density along the line of sight. Note that we cannot measure the RRM excess directly (see Section 3.2); however, we have shown that there are no indications of GRM differences between the samples and can therefore use the $|\mathrm{RM}|$ excess, $\Delta \mathrm{RM}$, as a proxy.

For the DLAs, given our data, there is a $32.1 \%$ probability that the $|\mathrm{RM}|$ is larger in the DLAs than in the controls. There is therefore little indication of enhanced coherent magnetic fields in the DLAs. If there were an increase, we calculate that there is a $90 \%$ probability that any increase over the control is, in $|\mathrm{RM}|$, $<1.88 \mathrm{rad} \mathrm{m}^{-2}$ in the observing frame. For our sample, we have a median $\log [N(\mathrm{H} \mathrm{I})]=20.63$ and a median DLA redshift of 1.947 . We therefore estimate, with $90 \%$ probability (given our data), that the regular coherent magnetic fields within the DLAs must be $\leqslant 2.8 \mu \mathrm{G}$. This estimate depends on the redshift range of the data, which varies between $z=0.53-2.22$. This corresponds to magnetic field upper limits of $\leqslant 0.8-3.3 \mu \mathrm{G}$. The estimate further depends on the $N(\mathrm{HI})$ range of the data, which varies between $2 \times 10^{20}-5 \times 10^{21}$ (by a factor of 25 ). Using the 25 th and 75 th percentiles, this would then correspond to magnetic field upper limits of $\leqslant 1.7-11.8 \mu G$.

For the LLSs, given our data, there is a $71.5 \%$ probability that the $|\mathrm{RM}|$ is larger in the LLSs than in the controls, although we note that we are unable to fully control for the redshift distribution of our data (see Section 3.4.5). There is therefore only a mild suggestive indication of enhanced coherent magnetic fields in the LLSs. We calculate that there is a $90 \%$ probability that any increase over the control is, in $|\mathrm{RM}|,<10.4 \mathrm{rad} \mathrm{m}^{-2}$ in the observing frame. For our sample, we have a median $\log [N$ $(\mathrm{HI})]=18.58$ and a median LLS redshift of 1.082 . We therefore estimate, with $90 \%$ probability (given our data), that the regular coherent magnetic fields within the LLSs must be $\leqslant 2.4 \mu \mathrm{G}$. This estimate depends on the redshift range of the data, which varies between $z=0.62-3.27$. This corresponds to magnetic field upper limits of $\leqslant 1.4-9.9 \mu G$. The estimate further depends on the $N(\mathrm{HI})$ range of the data, which varies between $1 \times 10^{17}-2 \times 10^{20}$ (by a factor of 1995). Using the 25 th and 75 th percentiles, this would then correspond to magnetic field upper limits of $\leqslant 0.8-33 \mu \mathrm{G}$. However, this difference could be attributed to the uncertainty on the RMs, which are of the order of $10 \mathrm{rad} \mathrm{m}^{-2}$. Future observations with increased RM precision will be able to improve upon these estimates.

One could argue that an alternative averaging procedure would be more appropriate, whereby we calculate the magnetic field in each absorber individually, and instead average the values of $\left\langle B_{\|}\right\rangle$. This would better incorporate the very large ranges in $N(\mathrm{H} \mathrm{I})$ and the variation arising from the factor $(1+z)^{2}$. Re-averaging provides updated mean estimates for coherent fields in DLAs to $\leqslant 2.8 \mu \mathrm{G}$ (unmodified from the original estimate) and in LLSs to $\leqslant 47.2 \mu \mathrm{G}$, and due to significant scatter provides median estimates in DLAs of $\leqslant 2.1 \mu \mathrm{G}$ and in LLSs of $\leqslant 1.6 \mu \mathrm{G}$. However, this alternative averaging does not include variations in $\bar{x}$ or, crucially, RRM between sources. As we have measured an ensemble average of RRM, we believe it more correct to provide an ensemble average of $\left\langle B_{\|}\right\rangle$rather than averaging over $\left\langle B_{\|}\right\rangle$, and therefore rely on our former estimates.

These prior upper limits are magnetic field constraints on the ISM of the DLAs/LLSs themselves. In the case of the DLAs, there may also be ionized material surrounding the high-density (mostly neutral) material, in a more highly ionized halo gas component. For example, C IV properties imply a ubiquitous, highly ionized, and enriched medium that traces the environments surrounding DLAs (Rubin et al. 2015). These C IV halos could give rise to Faraday rotation associated with the DLAs, even if the high column density component of the DLA itself is free of ionization. Following Rubin et al. (2015), the high incidence of strong C IV absorption and the large scales over which it is distributed point to a substantial reservoir of metals in the diffuse material surrounding DLAs at $z \sim 2$. A typical column density for C IV in DLAs is $N(\mathrm{C} \mathrm{IV})=10^{14} \mathrm{~cm}^{-2}$ (Fox et al. 2007), and a conservative ionization fraction is $\bar{x}(\mathrm{C}$ IV $)=0.3$ (Fox et al. 2007), which has been demonstrated to be the maximum possible ionization fraction in models assuming either photo- or collisional ionization. Taking there to be a $90 \%$ probability that any increase in Faraday rotation of the DLAs over the controls is, in $|\mathrm{RM}|,<1.88 \mathrm{rad} \mathrm{m}^{-2}$, and assuming that all of this Faraday rotation occurs in a C IV halo, then this places a very weak and uninformative constraint on the coherent magnetic field strength of $\leqslant 1.5 \mathrm{G}$ (note this is $\mathrm{G}$, rather than $\mu \mathrm{G})$. This is a magnetic field constraint for the typical halo surrounding a DLA. If we were to have detected an $|\mathrm{RM}|$ enhancement from DLAs, or if an enhancement is detected in the future, then it will be important to rule out whether the CIV halos surrounding DLAs could be responsible.

Given no information on the ionization state of the DLAs or the LLS, the Faraday rotation measurements can also serve as an indicator of the ionization fraction of these systems. Making a reasonable assumption of a universal coherent magnetic field strength of $1 \mu \mathrm{G}$ within the absorber, then this is consistent with a $90 \%$ probability that the DLA ionization fraction is $\bar{x} \leqslant 12.7 \%$ and that the LLS ionization fraction is $\bar{x} \leqslant 97.8 \%$.

\subsection{Random Magnetic Fields and Turbulence}

Foreground intervening screens that contain ionized gas and turbulent magnetic fields are known to depolarize a background 
Table 5

Results of the Robustness Checks on Calculated $p\left(\mathrm{RM}_{A}>\mathrm{RM}_{B}\right), p\left(\mathrm{GRM}_{A}>\mathrm{GRM}_{B}\right)$, and $p\left(\mathrm{E}\left[\Pi_{A}\right]<\mathrm{E}\left[\Pi_{B}\right]\right)$ for the Control, DLA, and LLS Samples

\begin{tabular}{|c|c|c|c|c|c|c|}
\hline Probability & $\begin{array}{c}\text { Main Results } \\
\%\end{array}$ & $\begin{array}{c}\text { Prior Selection } \\
\%\end{array}$ & $\begin{array}{c}\text { Non-intervenors } \\
\%\end{array}$ & $\begin{array}{l}\text { Spectral Dist. } \\
\%\end{array}$ & $\begin{array}{c}\text { Size Dist. } \\
\%\end{array}$ & $\begin{array}{c}z \text { Dist. } \\
\%\end{array}$ \\
\hline$\overline{\sigma_{\mathrm{RM}}(\mathrm{DLA})>\sigma_{\mathrm{RM}}(\text { control })}$ & 32.1 & 30.8 & 43.3 & 43.0 & 35.8 & 17.4 \\
\hline$\sigma_{\mathrm{RM}}(\mathrm{LLS})>\sigma_{\mathrm{RM}}($ control $)$ & 99.0 & 99.0 & 99.1 & 99.7 & 99.3 & 71.5 \\
\hline$\sigma_{\mathrm{RM}}(\mathrm{LLS})>\sigma_{\mathrm{RM}}(\mathrm{DLA})$ & 97.1 & 97.2 & 95.0 & 97.0 & 97.1 & 87.8 \\
\hline$\sigma_{\mathrm{GRM}}(\mathrm{DLA})>\sigma_{\mathrm{GRM}}($ control $)$ & 38.2 & 34.2 & 48.8 & 51.7 & 43.7 & 36.0 \\
\hline$\sigma_{\mathrm{GRM}}(\mathrm{LLS})>\sigma_{\mathrm{GRM}}($ control $)$ & 39.8 & 39.5 & 46.0 & 55.6 & 46.3 & 46.0 \\
\hline$\sigma_{\mathrm{GRM}}(\mathrm{LLS})>\sigma_{\mathrm{GRM}}(\mathrm{DLA})$ & 52.6 & 56.0 & 48.2 & 52.7 & 52.7 & 58.2 \\
\hline $\mathrm{E}[\Pi(\mathrm{DLA})]<\mathrm{E}[\Pi$ (control) $]$ & 45.2 & 36.5 & 40.5 & 42.0 & 44.6 & 34.7 \\
\hline $\mathrm{E}[\Pi($ LLS $)]<\mathrm{E}[\Pi$ (control) $]$ & 95.5 & 94.6 & 94.6 & 94.4 & 95.2 & 91.2 \\
\hline $\mathrm{E}[\Pi(\mathrm{LLS})]<\mathrm{E}[\Pi(\mathrm{DLA})]$ & 90.0 & 91.6 & 90.3 & 90.1 & 90.1 & 90.5 \\
\hline
\end{tabular}

Note. For clarity, our main results from Tables 3 and 4 are reproduced in the "Main Results" column.

source (Burn 1966; Sokoloff et al. 1998; Stil et al. 2009). Such depolarization manifests as a reduction in the observed polarized fraction. We can therefore use our measurements of the polarized fractions associated with the absorption line systems, relative to the control sample, in order to place constraints on the random magnetic fields, the ionized gas distribution, and the degree of turbulence in the DLAs and the LLSs.

Following Burn (1966), for the case of external Faraday dispersion by a non-emitting screen that contains thermal electrons and turbulent magnetic fields, but does not contain cosmic-ray electrons, the depolarization is given by

$$
\Pi=\Pi_{0} e^{-2 \sigma_{\mathrm{RM}}^{2} \lambda^{4}}
$$

where $\sigma_{\mathrm{RM}}$ is the RM dispersion from the foreground screen(s), and $\Pi$ and $\Pi_{0}$ are the observed and intrinsic polarized fractions, respectively, and $\lambda$ is the observing frequency. In principle, the Faraday screen needs to be corrected to cosmological distances by including a $(1+z)^{4}$ term:

$$
\Pi=\Pi_{0} e^{-2 \sigma_{\mathrm{RM}}^{2}(1+z)^{-4} \lambda^{4}},
$$

However, this term is defined in the case of a non-evolving screen (Bernet et al. 2012). Evolving screens are expected for magnetic fields that change via the dynamo mechanism. One simple physical model for Ly $\alpha$ clouds is $\sigma_{\mathrm{RM}}^{2} \propto(1+z)^{4}$ (Kronberg \& Perry 1982), which leads to complete cancellation of the $(1+z)^{4}$ term. ${ }^{8}$ The rest-frame polarized fractions also cannot be calculated from our current data, as we lack the broadband polarized spectral energy distributions that are required for $K$-correction and which will become available in future studies (Farnes et al. 2014a). We therefore remain in the observing frame for our calculations. Future studies will be able to attempt a reformulation of the theory when applying, e.g., non-Burn-style depolarization laws; however, such theoretical considerations are well beyond the scope of this paper.

\footnotetext{
8 In the standard dynamo, a small-scale magnetic field with a mean value tending to zero is able to generate a weak large-scale field. The maximum scatter in RM, $\sigma_{\mathrm{RM}}$, at any cosmological epoch is therefore proportional to the maximum magnetic field strength at that redshift, i.e., $\sigma_{\mathrm{RM}} \propto\left\langle B_{\|}\right\rangle$. In this way, this possible model of coherent field evolution in Ly $\alpha$ clouds can predict to first order the evolution of the turbulent component of the field.
}

We wish to compare a sample, $k$, which contains lines of sight with both an intrinsic and an intervening screen, such that

$$
\sigma_{\mathrm{RM}, k}^{2}=\sigma_{\mathrm{RM}, \text { intrinsic }}^{2}+\sigma_{\mathrm{RM}, \text { interv }}^{2},
$$

with a control, $j$, which contains lines of sight with only an intrinsic screen, such that

$$
\sigma_{\mathrm{RM}, j}^{2}=\sigma_{\mathrm{RM}, \text { intrinsic }}^{2}
$$

Since $\Pi_{0}$ is the intrinsic polarized fraction of the background QSOs, it can therefore be trivially shown that

$$
\ln \left(\frac{\Pi_{j}}{\Pi_{k}}\right)=2 \sigma_{\mathrm{RM}, \text { interv }}^{2} \lambda^{4} .
$$

Following Arshakian \& Beck (2011), the RM dispersion can be described in a simplified model of a turbulent magnetoionic medium as

$$
\sigma_{\mathrm{RM}}^{2} \simeq\left(0.81\left\langle n_{e}\right\rangle\left\langle B_{\mathrm{turb}}\right\rangle\right)^{2} \frac{L d}{f},
$$

where $n_{e}$ is the electron density in $\mathrm{cm}^{-3}$ within the turbulent cells, $\left\langle n_{e}\right\rangle$ is the average electron density in the volume along the path length traced by the telescope beam, $L$ is the path length in parsec, $d$ is the size of the turbulent cells (also called the correlation length) in parsec, $f$ is the filling factor of the cells given by $f=\left\langle n_{e}\right\rangle / n_{e}$, and $\left\langle B_{\text {turb }}\right\rangle$ in $\mu \mathrm{G}$ is the mean strength of the turbulent magnetic field, assumed to be the same both inside and outside of the cells. In particular, the characteristic scale of turbulence, $d$, the scale at which the dominant source of turbulence injects energy into the ISM of an LLS or DLA, is a key parameter to characterize magnetic turbulence in the associated gas. Finally it can be shown by combining Equations (13) and (14) that

$$
\frac{1}{2 \lambda^{4}} \ln \left(\frac{\Pi_{j}}{\Pi_{k}}\right) \simeq\left(0.81\left\langle n_{e}\right\rangle\left\langle B_{\text {turb }}\right\rangle\right)^{2} \frac{L d}{f},
$$

where all of the physical properties now parameterize the intervening absorption system, i.e., a DLA or LLS.

For the LLSs, we found a $95.5 \%$ probability that there is a decrease in the polarized fraction relative to the control sample. From Prochaska (1999), we use the LLS-derived estimates for the electron density $=6.5 \pm 1.3 \times 10^{-2} \mathrm{~cm}^{-3}$ and the path length $=3 \pm 1.6 \mathrm{kpc}$, and assume a filling factor of 1 . We previously obtained median polarized fractions of $4.2 \%$ for the controls and $3.3 \%$ for the LLSs, as observed at $1.4 \mathrm{GHz}$. This 
difference clearly cannot be attributed to the uncertainty on the polarized fractions, which are only of the order of $0.1 \%$. We therefore derive $d\left\langle B_{\text {turb }}\right\rangle^{2}=7.34 \mathrm{pc} \mu \mathrm{G}^{2}$. This is equivalent to a typical turbulent scale of $7.34 \mathrm{pc}$ in the LLSs for a turbulent magnetic field strength of $1 \mu \mathrm{G}$. However, we could realistically expect weaker turbulent fields: in Section 4.2 we estimated with $90 \%$ probability that the regular coherent magnetic fields within the LLSs must be $\leqslant 2.4 \mu \mathrm{G}$. If the random and coherent field components were of similar strength, this would increase the estimated turbulent scale to be on the order of $\approx 17 \mathrm{pc}$ or larger. To summarize, to be consistent with the data and realistic expectations for the magnetic field, the LLSs must have a model-dependent turbulent scale on the order of $\approx 5-20 \mathrm{pc}$. This is similar to the characteristic outer scale of turbulence seen in normal galaxies and is consistent with the case of, e.g., galaxy NGC 6946, for which a turbulent scale of $20 \pm 10$ pc was estimated by Beck et al. (1999) and is also in good agreement with the turbulent scale derived toward the Fan region in the Milky Way (Iacobelli et al. 2013). LLSs are predicted to have very little star formation (Erkal 2015) and yet turbulence is known to be an important contributor to star formation (Herron et al. 2017). Given the different nature of the ISM in LLSs compared to NGC 6946 and the Milky Way, the different star formation properties and matching turbulent scales are therefore quite surprising. We therefore interpret the matching turbulent scale as being somewhat fortuitous. The depolarization could also be conjectured to be the result of a patchy ionized medium in the LLSs, which serves as a depolarizing screen, in which case our estimates would constrain the physical size of the ionized clumps. However, the known high ionization fraction of $\bar{x} \approx 95 \%$ would appear to be inconsistent with this interpretation. We interpret our data as showing that an incoherent magnetic field must be present and that the magnetized gas in LLSs must be highly turbulent.

For the DLAs, we found a $45.2 \%$ probability that there is a decrease in the polarized fraction relative to the control sample. Depolarization by the DLAs therefore seems unlikely. However, if there is a decrease, we calculate a $90 \%$ probability that any decrease relative to the control must be $\leqslant 0.80 \%$. Using similar parameters to those derived in Prochaska (1999), we calculate an upper limit for the DLAs, and a $90 \%$ probability that $d\left\langle B_{\text {turb }}\right\rangle^{2} \leqslant 6.52 \mathrm{pc} \mu \mathrm{G}^{2}$. Depolarization can be caused by variations in the magnetic field, or in the thermal electron density. Due to self-shielding and the low ionized fraction, we do not expect any significant spatial variations in free electrons within the DLA environment. We interpret this to mean that there must be little variation in magnetic field within the DLAs. This must indicate either (i) that the disordered magnetic field strength is exceptionally weak, so that variations leave no measurable signal, or (ii) that the magnetic field must be highly coherent. Given the presumed young, dense, and clumpy form of the DLAs, together with our prior limits on the coherent magnetic field strength (see Section 4.2), this favors the former interpretation. The weak random magnetic field strength suggests that the magnetized gas in DLAs is non-turbulent and quiescent.

\subsection{The Dynamo Paradigm}

Previous studies have been able to reliably observe the magnetized environment of strong Mg II absorbers (e.g., Farnes et al. 2014b). We have now analyzed the magnetic fields in two new types of absorption line systems, the DLAs and the LLSs, using modern polarimetric data. The magnetic properties of DLAs have only ever provided tentative results using three to five sources (see Section 1), and in particular the magnetic environment of LLSs has never been studied before. It is therefore now of interest to consider the precise physical structures that are being probed at different stages of galaxy formation history and to examine the constraints that are emerging for dynamo mechanisms (e.g., Rodrigues et al. 2015). It is of specific interest whether dynamos can generate coherent-enough fields to contribute toward Faraday rotation and depolarization effects (Bhat \& Subramanian 2013).

From Pakmor et al. (2014), it has previously been determined from cosmological simulations of the formation and evolution of galaxies that a prescribed tiny magnetic seed field grows exponentially through a small-scale dynamo until it saturates around $z=4$ with a magnetic energy of about $10 \%$ of the kinetic energy in the center of the galaxy's main progenitor halo. By $z=2$, a well-defined gaseous disk forms in which the magnetic field is further amplified by differential rotation, until it saturates at an average field strength of $\sim 6 \mu \mathrm{G}$ in the disk plane. During this latter stage, the magnetic field is transformed from a chaotic small-scale field to an ordered large-scale field coherent on scales comparable to the disk radius.

Similarly, Arshakian et al. (2009) used dynamo theory to show that turbulence in protogalactic halos (an environment related to the LLSs; see Section 1.1) generated by thermal virialization can drive an efficient turbulent dynamo, with the turbulent (small-scale) dynamo being able to amplify a weak seed magnetic field in halos of protogalaxies to a few $\mu \mathrm{G}$ strength within a few $10^{8}$ years. Consistent with the cosmological simulations, this turbulent field served as a seed to the mean-field (large-scale) dynamo. Consequently, Arshakian et al. (2009) found that galaxies similar to the Milky Way formed their disks at $z \approx 10$, and regular fields of $\mu \mathrm{G}$ strength and a few kpc coherence length were generated within $2 \mathrm{Gyr}$ (at $z \approx 3$ ). However, field ordering on the coherence scale of the galaxy size required an additional $6 \mathrm{Gyr}$ (at $z \approx 0.5$ ). Overall, this demonstrates that magnetic field generation by the turbulent dynamo in galaxies requires neither large-scale rotation nor a disk, only turbulence. In addition, high-resolution simulations of protogalactic clouds have also demonstrated that significant turbulence can be generated prior to disk formation during the thermal virialization of the halo (Wise \& Abel 2007). The big picture is then that strong turbulence in protogalactic halos at early epochs can drive the small-scale dynamo and amplify the seed field. This turbulent dynamo produces magnetic fields on scales comparable to the basic scale of galactic turbulence on timescales far shorter than that for the conventional mean-field galactic dynamo (e.g., Batchelor 1950). In the epoch of disk formation, the turbulent field then served as a seed for the largescale dynamo that develops in the disk of a newly formed galaxy.

Our data appear fully consistent with this conventional dynamo paradigm. We interpret our data as showing that the LLSs mostly consist of turbulent gas, while the DLAs mostly consist of quiescent gas. In the LLSs, we have found that turbulence has increased the random magnetic field, but not the coherent field. While we only have two limits on the coherent field strength in both galaxy types, these limits are, however, quite constraining: $\leqslant 2.8 \mu \mathrm{G}$ in DLAs and $\leqslant 2.4 \mu \mathrm{G}$ in LLSs. There are therefore no indications, within the sensitivity of 
these observations, that the coherent fields have been amplified via the dynamo mechanism. It is the limits that allow us to make this claim: if the coherent fields had increased, we would instead expect a detectable enhancement in $|\mathrm{RM}|$. The correspondence with the expectations of dynamo theory therefore seems unlikely to be a mere coincidence. However, we highlight that the presence of coherent fields may be just below our ability to detect them with these data (see Section 3.4.5). With no detectable increase in the coherent field, this is consistent with action from the small-scale dynamo, and with there not having been sufficient time for either disk formation or driving the large-scale dynamo. This has resulted in no, or a very limited, large-scale field in LLSs. In contrast, in the DLAs, the gas appears to be mostly quiescent, and DLAs must have very weak non-detectable magnetic fields in both their coherent and random components. This is consistent with no detectable large-scale or small-scale magnetic field. This demonstrates limited, or a complete lack of, dynamo action in DLAs (see also Section 4.5).

Our findings imply an evolutionary hierarchy, with different absorption lines probing varying stages of galaxy formation. The lack of a magnetic environment observed in DLAs, the random magnetic fields in LLSs, and the strong coherent fields observed in previous studies of strong Mg II absorbers (e.g., Farnes et al. 2014b) all suggest that DLAs probe less-evolved galaxies than LLSs, and similarly that LLSs may be lessevolved counterparts to the strong Mg II absorbers. In this hierarchical scenario, the strong $\mathrm{Mg}$ II absorbers have had enough time to have driven the large-scale dynamo and to generate a coherent field. We are therefore exploring three different stages of magnetic field evolution. Notably, the only strong Mg II absorbers measured to date have been seen at lower redshifts of $z \approx 1$, and are typically associated with normal star-forming galaxies (e.g., Farnes et al. 2014b), many of which have kinematics consistent with a disk/halo structure. In contrast, the to-date unstudied (in terms of magnetic field properties) strong Mg II systems at higher redshifts of $z \approx 2$ are known to be associated with protogalactic structures (Mshar et al. 2007). This would suggest that future studies of higher redshift strong $\mathrm{Mg}$ II absorbers may identify similar properties to those of the LLSs. This may hinder the use of QSOabsorption line systems for exploring the dynamo out to higher redshifts, in cases where the physical nature of the absorption line system evolves with time. The first step in this process will be further studies where we use Lyman systems out to $z \approx 3$ and assume these will evolve into normal galaxies by $z \approx 0$, which is consistent with hierarchical galaxy formation evidenced by deep galaxy surveys. Multifaceted studies that utilize many absorption lines and explore the physical environment associated with each absorption line type will be key to charting the evolution of magnetic fields throughout cosmic time.

\subsection{Measuring the Magnetized Large-scale Structure and Seed Fields}

We have shown that the gas in DLAs is mostly quiescent, and that DLAs lack a detectable large-scale or small-scale magnetic field. This is consistent with no dynamo action having taken place in these protogalactic clumps. This implies that DLAs may therefore form natural repositories through which we can measure the magnetic field and infer the magnetic properties in the large-scale structure of the intergalactic medium (IGM; e.g., Akahori \& Ryu 2010) and even possibly of magnetic seed fields. Observations with substantially larger samples and refined RM data via techniques such as $Q U$-fitting (e.g., O'Sullivan et al. 2012) may be able to place tighter limits on these weak fields. However, direct detection of seed fields remains a very distant prospect. Observing a seed magnetic field of $10^{-6} \mu \mathrm{G}$ requires the ability to detect a $\triangle \mathrm{RRM}=0.66 \mu \mathrm{rad} \mathrm{\textrm {m } ^ { - 2 }}$. The signal of interest is the difference in the standard deviation of the RM distributions of the DLA, LLS, and control samples, where the intrinsic scatter due to QSO variations is $\sim 15 \mathrm{rad} \mathrm{m}^{-2}$. To first order, assuming we can calculate the standard deviation in RM to within an uncertainty of $\sigma / \sqrt{2(n-1)}$, we would require samples of $10^{15}$ polarized sources in order to measure such a signal. Such measurement is evidently beyond reach of even the SKA, for which $10^{7}$ RM measurements are expected during SKA phase 1 (Johnston-Hollitt et al. 2015). Such a measurement would also require substantial leaps in other areas: in the number of known DLAs, in Galactic RM foreground subtraction, and in the precision of RM measurements.

However, DLAs may also represent intermediate stages of evolution that lie somewhere between a dynamo-free clump and a small-scale dynamo-fueled LLS. This is highly likely as a DLA should have frozen in the magnetic fields from the IGM into its denser medium. In this case, the DLAs may still have turbulence and reasonable strength magnetic fields that are greater than a typical seed field and possibly detectable with upcoming instruments. Such fields are currently below our detection threshold. Further data will be able to improve our estimates of the magnetic field in these objects.

\section{Conclusions and Summary}

We have studied samples of QSOs with intervening DLAs or LLSs identified somewhere along their line of sight, and compared these samples to a control sample of QSOs with no intervening DLAs or LLSs. We have compared these data using a Bayesian analysis, and it is the first time that such a statistical method has been applied to Faraday rotation and fractional polarization data in this way. The new application of this statistical tool opens a new method for quasar absorption line studies, in which many absorption lines have only a few quasar candidates and hence small sample sizes. This Bayesian analysis remains robust in the small sample regime and therefore opens up previously restrictively sized samples for analysis. This also presents a new way to more rigorously assess claimed correlations from previous studies of strong (e.g., Bernet et al. 2008; Farnes et al. 2014b) and weak (e.g., Kim et al. 2016) Mg II absorbers.

Given our data, we have found that:

1. It is unlikely that DLAs have coherent magnetic fields, with a $32.1 \%$ probability that DLA lines of sight have a higher $|\mathrm{RM}|$ than a control sample. It is also unlikely that DLAs have random magnetic fields, with a $45.2 \%$ probability that DLA lines of sight have a lower polarized fraction than a control sample.

2. There are mild suggestive indications that LLSs have coherent magnetic fields. Although we are unable to fully control for the redshift distribution of our data, when we attempt to do so, we find a $71.5 \%$ probability that LLS 
lines of sight have a higher $|\mathrm{RM}|$ than a control sample. However, it is extremely likely that LLSs have random magnetic fields, with a $95.5 \%$ probability that LLS lines of sight have a lower polarized fraction than a control sample.

3. We model our data to show that there is a $90 \%$ probability that the regular coherent magnetic fields within the DLAs must be $\leqslant 2.8 \mu \mathrm{G}$, and within the LLSs must be $\leqslant 2.4 \mu \mathrm{G}$.

4. We also model the turbulent magnetic fields and find that our data are most consistent with the DLAs having weak random magnetic fields, which suggest that the magnetized gas in DLAs is non-turbulent and quiescent. We also find that the LLSs must have an incoherent magnetic field present, and that the magnetized gas must be highly turbulent with a model-dependent turbulent scale on the order of $\approx 5-20 \mathrm{pc}$, which is similar to the measured turbulent scale within the Milky Way (likely coincidentally).

Overall, a clear picture consistent with typical expectations of dynamo evolution is beginning to emerge from these absorption line studies. Our data are entirely consistent with the conventional interpretation of DLAs as protogalaxies, combined with the paradigm that dynamo action acts on weak disordered magnetic fields to generate coherent strong fields. Such studies hold promise for charting out dynamo evolution over cosmic time. Understanding the environment of different physical systems such as DLAs, LLSs, and other absorption lines will be the first step in beginning to directly map the evolution of cosmic magnetism. Using the combination of strong Mg II absorbers, LLSs, and DLAs, we have begun to chart out magnetic fields out to $z \approx 2$. Our data allow us to see the first observational picture of magnetic field evolution in galaxies. Protogalaxies that lack a coherent magnetic field, and which maintain significant random magnetic fields, are evolving into normal star-forming galaxies with strong coherent fields. This reaffirms the role that magnetic fields play in the formation and evolution of galaxies. This is also the first time that the magnetic fields of LLSs have ever been studied, and the first time that DLAs or LLSs have been shown to have different magnetic field properties from those of a control sample. This also allows us to conclude that the seeding of magnetic fields by supernovae and subsequent amplification during structure formation are able to build up strong magnetic fields of $\mu \mathrm{G}$ strength within short time spans. This leads to detectable magnetic fields within the very first collapsing and star-forming protohalos at high redshifts, which are the building blocks for the very first galaxies.

The developed techniques can also allow us to slowly push back toward the seed field and magnetogenesis era. Seed fields could have been generated in protogalaxies in the early universe, e.g., at phase transitions or in shocks in protogalactic halos (via the Biermann battery), or in fluctuations in the protogalactic plasma. Nevertheless, these seed fields will remain observationally out of reach for a long time and remain six orders of magnitude below current observational thresholds, and so are expected to remain undetectable even with the SKA. However, with the introduction of a more refined Bayesian analysis for such studies, this work has created a quantitative gateway toward accessing and constraining magnetic fields in the IGM via quasar absorption line experiments. Future studies will be able to use the lowest column density Lyman systems, the Ly $\alpha$ forest at $\leqslant 10^{17} \mathrm{~cm}^{-2}$, in order to constrain magnetism in the IGM.

We are currently lacking a large sample size in our study. This prevents us from subdividing the sample further, in order to explore other connections between the absorbers based on redshift, column density, and other intervenor parameters. In the future, with larger samples of sources with high polarized signal-to-noise ratio so as not to be affected by Rician bias, we will be able to explore evolution based on the redshift of the absorbers to definitively rule out evolution based on the redshift of the quasars and to explore the evolution in magnetic and ionization properties based on column density alone. Other subdivisions, such as the spectral index, are also of importance and has been shown to be important for studies of strong $\mathrm{Mg}$ II absorbers. Nevertheless, the spectral index is far less important for the DLAs and LLSs, which are known to be smoothly distributed with a higher covering fraction compared to the clumpy partially ionized medium with low-covering fraction that is associated with strong $\mathrm{Mg}$ II. It is therefore to be expected that the magnetic environment extends to larger scales in the DLAs and LLSs than in the Mg II absorbing systems. Furthermore, the DLA and LLS samples also include a very tight cross-matching criterion of 2 arcsec, which is equal to $25 \mathrm{kpc}$ at $z=1$, and therefore less than typical estimates for the DLA/LLS size. Increased sample sizes with higher angular resolution will allow us to further subdivide our data and to test these attributes. Future studies will therefore be able to subdivide the data into flat and steep samples with high angular resolution, allowing for direct tests of the requirement for single-component compact sources and also providing a unique way to measure the ionized size of these absorbers. Future data that will become available as DLAs and other absorption features are identified in the SDSS DR10 or DR12 will also improve our sample size and allow for further subdivision into smaller subpopulations (Pâris et al. 2017; Raghunathan et al. 2016). In addition, upcoming radio data from, e.g., the Very Large Array Sky Survey ${ }^{9}$ (VLASS), have the potential to identify sightlines for Faraday Tomography through as many as 40,000 Mg II absorbers.

Finally, future studies will also be able to refine the Bayesian analysis of Faraday rotation data introduced here for quasar absorption lines. Testing other distributions, alongside the normal distributions assumed in this paper, will provide improvements and will refine future results with larger samples. Such an analysis will most likely be unable to use conjugate priors and so will require more sophisticated sampling techniques to be used. The use of hierarchical Bayesian methods will help to further refine our methodology (e.g., Vacca et al. 2016) and future experiments can be devised in order to allow for tests of mutual consistency between data sets (Karpenka et al. 2015). We also discuss our model selection in the Appendix. If there is substantial deviation from these model choices, then there would be implications for most previous magnetic field studies throughout the literature, as our chosen models have often been used either explicitly via model fitting or the calculation of standard deviations, or implicitly via the use of frequentist tests. While we have been able to model the coherent magnetic fields using the RMs and GRMs, further analysis of the distribution of these statistical samples will allow us to ensure that our implicit assumption, that

\footnotetext{
9 https://safe.nrao.edu/wiki/pub/JVLA/VLASS/VLASS_final.pdf
} 
$\sigma_{\mathrm{RM}}^{2}=\sigma_{\mathrm{GRM}}^{2}+\sigma_{\mathrm{RRM}}^{2}$ (see Section 3.2), is correct. Expansion of our Bayesian technique may also allow us to incorporate modeling of the error terms that currently inhibit use of RRMs, and thereby allow a statistical measurement with the RRMs directly. While this could have implications for our coherent magnetic field estimates in both DLAs and LLSs, our primary result of depolarization associated with the LLSs would of course remain unaffected. The application of these Bayesian techniques offers the opportunity to remove the systematic effects that frequently affect correlation-based studies and to provide a thorough rigorous framework for testing connections between other absorption line systems.

We are grateful to the anonymous referee, to Niels Oppermann, and to Mike Hobson, for useful comments that improved the paper. The National Radio Astronomy Observatory is a facility of the National Science Foundation operated under cooperative agreement by Associated Universities, Inc. This research has made use of the SDSS, for which funding has been provided by the Alfred P. Sloan Foundation, the Participating Institutions, the National Science Foundation, and the U.S. Department of Energy Office of Science. Partial support for this research at the University of Minnesota was provided by U.S. National Science Foundation Grant AST1211595. B.M.G. acknowledges the support of the Natural Sciences and Engineering Research Council of Canada (NSERC) through grant RGPIN-2015-05948. The Dunlap Institute is funded through an endowment established by the David Dunlap family and the University of Toronto. S.P.O. acknowledges support from UNAM through the PAPIIT project IA103416.

\section{Appendix \\ Bayesian Analysis}

\section{A.1. Analysis of Faraday Rotation}

We model the distribution of RMs and GRMs with Gaussian distributions centered at $0 \mathrm{rad} \mathrm{m}^{-2}$. Using the RMs and GRMs, we therefore wish to model the joint posterior distribution of both the mean, $\mu$, and variance, $\sigma^{2}$, given the data, $D$, for which the posterior is given by $p\left(\mu, \sigma^{2} \mid D\right)$.

We apply Bayes' theorem, using the notation of Gelman et al. (2004) throughout,

$$
p\left(\mu, \sigma^{2} \mid D\right)=\frac{p\left(D \mid \mu, \sigma^{2}\right) p\left(\mu, \sigma^{2}\right)}{p(D)},
$$

where $p\left(D \mid \mu, \sigma^{2}\right)$ is the Gaussian likelihood function, $p(D)$ is purely a function of the data, and $p\left(\mu, \sigma^{2}\right)$ is the joint prior distribution. The probability of the data, $p(D)$, is just a normalization constant and does not affect our results.

Following Gelman et al. (2004), we choose a conjugate prior distribution for the two-parameter univariate normal sampling model, as this has the advantage that it is analytically tractable. The conjugate prior is of the form $p\left(\mu, \sigma^{2}\right)=p\left(\sigma^{2}\right) p\left(\mu \mid \sigma^{2}\right)$, where $p\left(\sigma^{2}\right)$ is a scaled inverse- $\chi^{2}$ distribution and $p\left(\mu \mid \sigma^{2}\right)$ is a normal distribution. This corresponds to the joint prior distribution

$$
\begin{aligned}
p\left(\mu, \sigma^{2}\right) \propto & \sigma^{-1}\left(\sigma^{2}\right)^{-\left(1+\nu_{0} / 2\right)} \exp \\
& \times\left(-\frac{1}{2 \sigma^{2}}\left[\nu_{0} \sigma_{0}^{2}+\kappa_{0}\left(\mu_{0}-\mu\right)^{2}\right]\right),
\end{aligned}
$$
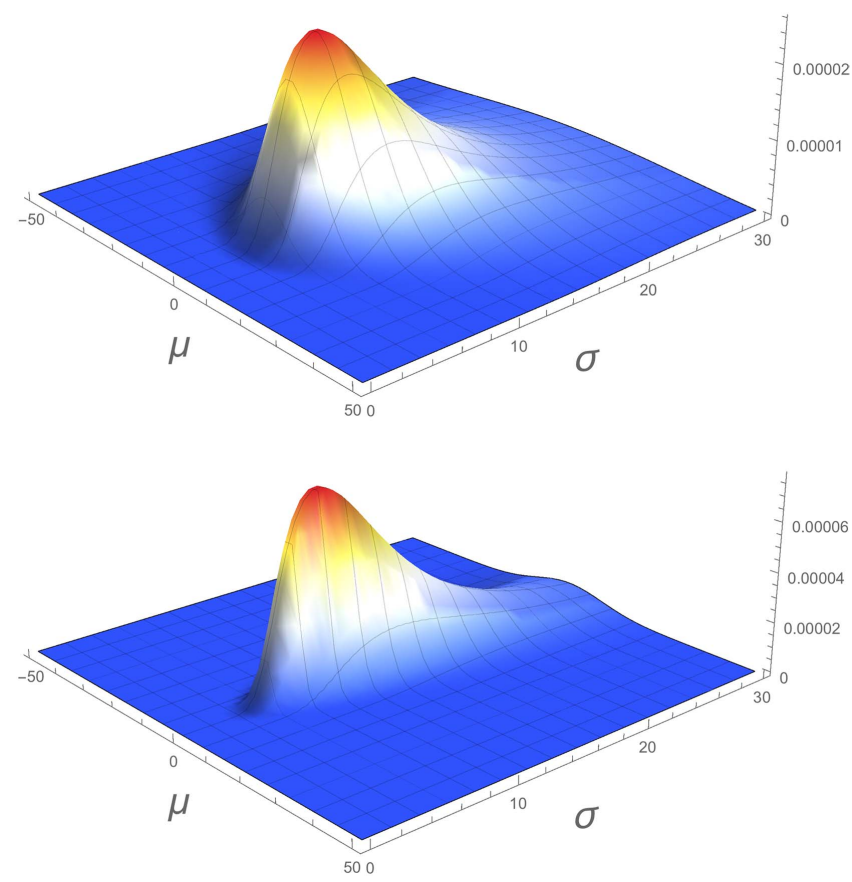

Figure 11. Selected priors used for the Faraday rotation estimates. Both the RM (top) and GRM (bottom) priors are shown. $\mu$ and $\sigma$ both have units of $\mathrm{rad} \mathrm{m}^{-2}$. Both priors are weakly informative, and we confirm that we are not sensitive to the choice of prior in Section 3.4.

where the four hyperparameters can be identified, in simple terms, as $\mu_{0}$-the prior mean, $\kappa_{0}$-our degree of belief in this parameter (which is also equivalent to the number of prior measurements, while the scale of $\mu_{0}$ is given by $\left.\sigma_{0}^{2} / \kappa_{0}\right), \sigma_{0}^{2}$-the prior variance, and $\nu_{0}$-our degree of belief in this parameter (equivalent to the degrees of freedom of $\sigma_{0}^{2}$ ). We choose our prior parameters for the RM to be weakly informative, setting $\mu_{0}=0.0 \mathrm{rad} \mathrm{m}^{-2}, \kappa_{0}=1.0, \sigma_{0}^{2}=25.0^{2} \mathrm{rad}^{2} \mathrm{~m}^{-4}$, and $\nu_{0}=$ 0.5 . For the GRM, we also use a weakly informative prior but include mildly stronger belief that the mean of the GRM is centered at $0.0 \mathrm{rad} \mathrm{m}^{-2}$, setting $\mu_{0}=0.0 \mathrm{rad} \mathrm{m}^{-2}, \kappa_{0}=10.0$, $\sigma_{0}^{2}=25.0^{2} \mathrm{rad}^{2} \mathrm{~m}^{-4}$, and $\nu_{0}=0.5$. The priors for the Faraday rotation estimates are shown in Figure 11.

\section{A.2. Analysis of Polarized Fractions}

We now consider the case of the polarized fraction values, $\Pi$, associated with the DLA and LLS. Unlike the RM and GRM values, the $\Pi$ values are likely not well-modeled by a normal distribution. As we know that the polarized fractions are positive-definite, and also that the distributions appear skewed toward zero (see Figure 8), we instead select a lognormal distribution as our model (e.g., Tucci \& Toffolatti 2012; Massardi et al. 2013). ${ }^{10}$

We therefore first take the logarithm of the data samples (as shown in Figure 8), and our approach is then similar to that used in Section 3.2 for normal data. Again, using a conjugate prior and a Gaussian likelihood, we specify posterior distributions of the parameters $\mu$ and $\sigma$. We then draw samples of $\mu$ and $\sigma$ from the posteriors and transform each of them back into non-logarithmic space. As we are interested in the arithmetic

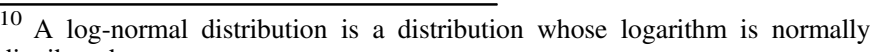
distributed.
} 


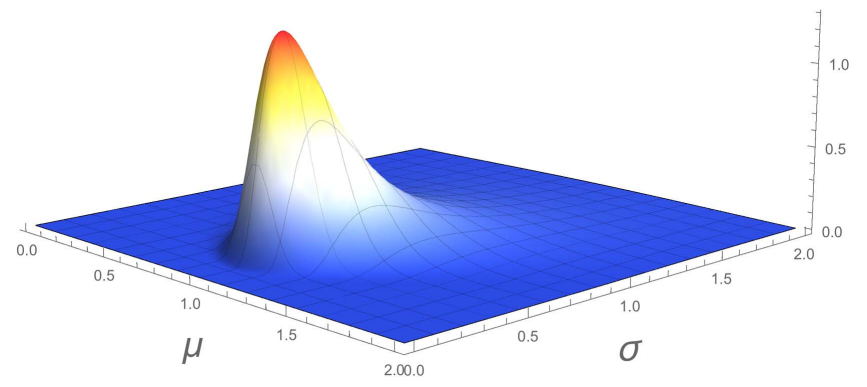

Figure 12. Selected prior used for the polarized fraction estimates. $\mu$ and $\sigma$ are both unitless. The prior is weakly informative, and we confirm that we are not sensitive to the choice of prior in Section 3.4.

mean and arithmetic standard deviation of each of the lognormal distributions, these are given by

$$
\mathrm{E}[\Pi]=e^{\mu+\frac{\sigma^{2}}{2}}
$$

and

$$
\mathrm{SD}[\Pi]=e^{\mu+\frac{\sigma^{2}}{2}} \sqrt{e^{\sigma^{2}}-1} .
$$

The full framework is otherwise done in the same manner as in Appendix A.1. We choose our prior parameters to be weakly informative, setting $\mu_{0}=1.0, \kappa_{0}=10.0, \sigma_{0}^{2}=1.0^{2}$, and $\nu_{0}=0.5$. $\mu$ and $\sigma$ are both unitless. The prior is shown in Figure 12.

\section{A.3. Drawing Samples from the Posterior}

Also following Gelman et al. (2004), as we have chosen a conjugate prior, we have a closed-form expression for the joint posterior distribution, which is given by

$$
p\left(\mu, \sigma^{2} \mid D\right)=\mathrm{N}-\mathrm{Inv}-\chi^{2}\left(\mu_{n}, \sigma_{n}^{2} / \kappa_{n} ; \nu_{n}, \sigma_{n}^{2}\right),
$$

where N-Inv- $\chi^{2}$ is the normal inverse- $\chi^{2}$ distribution.

The conditional posterior distribution, $p\left(\mu \mid \sigma^{2}, D\right)$, of $\mu$, given $\sigma$, is then proportional to the joint posterior distribution with $\sigma^{2}$ held constant, which is given by

$$
\mu \mid \sigma^{2}, D \sim \mathrm{N}\left(\mu_{n}, \sigma^{2} / \kappa_{n}\right),
$$

where $\mathrm{N}$ is the normal distribution, and while the marginal posterior distribution, $p\left(\sigma^{2} \mid D\right)$, of $\sigma^{2}$, is a scaled inverse- $\chi^{2}$, which is given by

$$
\sigma^{2} \mid D \sim \operatorname{Inv}-\chi^{2}\left(\nu_{n}, \sigma_{n}^{2}\right)
$$

In order to sample from the joint posterior distribution, we will first draw a $\sigma^{2}$ from the marginal posterior distribution of Equation (22) and then draw $\mu$ from the normal conditional posterior distribution of Equation (21), using the simulated value of $\sigma^{2}$. In practice, we will instead draw samples using the inverse- $\Gamma$ (Inv- $\Gamma$ ) distribution and using the identity for an $\operatorname{Inv}-\chi^{2}\left(\nu_{0}, \sigma_{0}^{2}\right) \equiv \operatorname{Inv}-\Gamma\left(\nu_{0} / 2, \nu_{0} \sigma_{0}^{2} / 2\right)$. We will sample $2,000,000$ times in order to generate our samples of $\mu$ and $\sigma$.

\section{A.4. Model Selection}

To quote George Box, "all models are wrong, but some are useful." Our model selection is justified as our data show direct similarity to these distributions and as the models we use have been widely employed in previous studies with larger sample sizes (Hammond et al. 2012; Tucci \& Toffolatti 2012; Massardi et al. 2013; Farnes et al. 2014a). If our assumed normal- or log- normal distribution is a very bad approximation, then this would affect the conclusions of our paper. More importantly, if our models are incorrect, then this would have wide implications for the conclusions of various papers across the literature.

In the future, with larger samples, we will be able to more directly test our models, to expand upon our Bayesian framework to include an error analysis (possibly allowing for use of RRMs), and to test the effects of assuming different model distributions. These more developed cases will likely not be able to use a conjugate prior and will require more sophisticated techniques for sampling from the posterior distribution.

\section{References}

Akahori, T., \& Ryu, D. 2010, ApJ, 723, 476

Arshakian, T. G., \& Beck, R. 2011, MNRAS, 418, 2336

Arshakian, T. G., Beck, R., Krause, M., \& Sokoloff, D. 2009, A\&A, 494, 21 Batchelor, G. K. 1950, RSPSA, 201, 405

Beck, A. M., Dolag, K., Lesch, H., \& Kronberg, P. P. 2013, MNRAS, 435, 3575

Beck, R., Berkhuijsen, E. M., \& Uyaniker, B. 1999, in Proc. Int. Conf., Plasma Turbulence and Energetic Particles in Astrophysics, ed. M. Ostrowski \& R. Schlickeiser (Kraków: Obserwatorium Astronomiczne, Uniwersytet Jagielloński), 5

Bernet, M. L., Miniati, F., \& Lilly, S. J. 2012, ApJ, 761, 144

Bernet, M. L., Miniati, F., \& Lilly, S. J. 2013, ApJL, 772, L28

Bernet, M. L., Miniati, F., Lilly, S. J., Kronberg, P. P., \& Dessauges-Zavadsky, M. 2008, Natur, 454, 302

Bhat, P., \& Subramanian, K. 2013, MNRAS, 429, 2469

Burn, B. J. 1966, MNRAS, 133, 67

Cen, R. 2012, ApJ, 748, 121

Chamandy, L., Shukurov, A., Subramanian, K., \& Stoker, K. 2014, MNRAS, 443, 1867

Chamandy, L., \& Taylor, A. R. 2015, ApJ, 808, 28

Churchill, C. W., Rigby, J. R., Charlton, J. C., \& Vogt, S. S. 1999, ApJS, 120,51

Condon, J. J., Cotton, W. D., Greisen, E. W., et al. 1998, AJ, 115, 1693

Curran, S. J., Webb, J. K., Murphy, M. T., et al. 2002, PASA, 19, 455

Djorgovski, S. G., Pahre, M. A., Bechtold, J., \& Elston, R. 1996, Natur, 382, 234

Erkal, D. 2015, MNRAS, 451, 904

Farnes, J. S., Gaensler, B. M., \& Carretti, E. 2014, ApJS, 212, 15

Farnes, J. S., O’Sullivan, S. P., Corrigan, M. E., \& Gaensler, B. M. 2014, ApJ, 795, 63

Fontana, A., Cristiani, S., D’Odorico, S., Giallongo, E., \& Savaglio, S. 1996, MNRAS, 279, L27

Fox, A. J., Ledoux, C., Petitjean, P., \& Srianand, R. 2007, A\&A, 473, 791

Fox, A. J., Ledoux, C., Petitjean, P., Srianand, R., \& Guimarães, R. 2011, A\&A, 534, A82

Fox, A. J., Petitjean, P., Ledoux, C., \& Srianand, R. 2007, A\&A, 465, 171

Fumagalli, M., O'Meara, J. M., \& Prochaska, J. X. 2016, MNRAS, 455, 4100

Fumagalli, M., Prochaska, J. X., Kasen, D., et al. 2011, MNRAS, 418, 1796

Gaensler, B. M., Beck, R., \& Feretti, L. 2004, NewAR, 48, 1003

Gardner, J. P., Katz, N., Hernquist, L., \& Weinberg, D. H. 1997, ApJ, 484, 31

Gardner, J. P., Katz, N., Hernquist, L., \& Weinberg, D. H. 2001, ApJ, 559, 131

Gelman, A., Carlin, J., Stern, H., \& Rubin, D. 2004, Bayesian Data Analysis (2nd ed.; London: Chapman and Hall)

Green, D. A. 2011, BASI, 39, 289

Guimarães, R., Petitjean, P., de Carvalho, R. R., et al. 2009, A\&A, 508, 133 Haehnelt, M. G., Steinmetz, M., \& Rauch, M. 1998, ApJ, 495, 647

Hammond, A. M., Robishaw, T., \& Gaensler, B. M. 2012, arXiv:1209.1438

Herron, C. A., Federrath, C., Gaensler, B. M., et al. 2017, MNRAS, 466, 2272

Howk, J. C., Wolfe, A. M., \& Prochaska, J. X. 2005, ApJL, 622, L81

Iacobelli, M., Haverkorn, M., Orrú, E., et al. 2013, A\&A, 558, A72

Johnston-Hollitt, M., Govoni, F., Beck, R., et al. 2015, in Proc. Advancing Astrophysics with the Square Kilometre Array (AASKA14), ed. T. L. Bourke et al. (Trieste: POS), 92

Jorgenson, R. A., Murphy, M. T., \& Thompson, R. 2013, MNRAS, 435, 482 Kacprzak, G. G., Churchill, C. W., Steidel, C. C., Murphy, M. T., \& Evans, J. L. 2007, ApJ, 662, 909

Karpenka, N. V., Feroz, F., \& Hobson, M. P. 2015, MNRAS, 449, 2405 
Katz, N., Weinberg, D. H., Hernquist, L., \& Miralda-Escude, J. 1996, ApJL, 457, L57

Kim, K. S., Lilly, S. J., Miniati, F., et al. 2016, ApJ, 829, 133

Kronberg, P. P., \& Perry, J. J. 1982, ApJ, 263, 518

Kronberg, P. P., Perry, J. J., \& Zukowski, E. L. H. 1992, ApJ, 387, 528

Lanzetta, K. M., Webb, J. K., \& Barcons, X. 1996, ApJL, 456, L17

Ledoux, C., Petitjean, P., \& Srianand, R. 2003, MNRAS, 346, 209

Lehner, N., Howk, J. C., Prochaska, J. X., \& Wolfe, A. M. 2008, MNRAS, 390, 2

Longair, M. S. 2011, High Energy Astrophysics (Cambridge: Cambridge Univ. Press)

Maller, A. H., Prochaska, J. X., Somerville, R. S., \& Primack, J. R. 2001, MNRAS, 326, 1475

Massardi, M., Burke-Spolaor, S. G., Murphy, T., et al. 2013, MNRAS, 436, 2915

Meiksin, A. A. 2009, RvMP, 81, 1405

Meiring, J. D., Lauroesch, J. T., Kulkarni, V. P., et al. 2009, MNRAS, 397, 2037

Meylan, G. 1995, QSO Absorption Lines (Berlin: Springer)

Mshar, A. C., Charlton, J. C., Lynch, R. S., Churchill, C., \& Kim, T.-S. 2007, ApJ, 669, 135

Noterdaeme, P., Ledoux, C., Petitjean, P., \& Srianand, R. 2008, A\&A, 481,327

Noterdaeme, P., Petitjean, P., Carithers, W. C., et al. 2012, A\&A, 547, L1

Noterdaeme, P., Petitjean, P., Ledoux, C., \& Srianand, R. 2009, A\&A, 505, 1087

Noterdaeme, P., Petitjean, P., Pâris, I., et al. 2014, A\&A, 566, A24

Okoshi, K., \& Nagashima, M. 2005, ApJ, 623, 99

Oppermann, N., Junklewitz, H., Greiner, M., et al. 2015, A\&A, 575, A118

Oppermann, N., Junklewitz, H., Robbers, G., et al. 2012, A\&A, 542, A93

Oren, A. L., \& Wolfe, A. M. 1995, ApJ, 445, 624

Osterbrock, D. E. 1989, Astrophysics of Gaseous Nebulae and Active Galactic Nuclei (Mill Valley, CA: Univ. Science Books) Research supported by the University of California, John Simon Guggenheim Memorial Foundation, University of Minnesota, et al.

O’Sullivan, S. P., Brown, S., Robishaw, T., et al. 2012, MNRAS, 421, 3300

Pakmor, R., Marinacci, F., \& Springel, V. 2014, ApJL, 783, L20

Pâris, I., Petitjean, P., Aubourg, É., et al. 2012, A\&A, 548, A66

Pâris, I., Petitjean, P., Ross, N. P., et al. 2017, A\&A, 597, A79

Péroux, C., Dessauges-Zavadsky, M., D’Odorico, S., Kim, T.-S., \& McMahon, R. G. 2007, MNRAS, 382, 177

Pontzen, A., Governato, F., Pettini, M., et al. 2008, MNRAS, 390, 1349

Prochaska, J. X. 1999, ApJL, 511, L71

Prochaska, J. X., Howk, J. C., O’Meara, J. M., et al. 2002, ApJ, 571, 693
Prochaska, J. X., Howk, J. C., \& Wolfe, A. M. 2003, Natur, 423, 57

Prochaska, J. X., O’Meara, J. M., Fumagalli, M., Bernstein, R. A., \& Burles, S. M. 2015, ApJS, 221, 2

Prochaska, J. X., O’Meara, J. M., Herbert-Fort, S., et al. 2006, ApJL, 648, L97

Prochaska, J. X., O’Meara, J. M., \& Worseck, G. 2010, ApJ, 718, 392

Prochaska, J. X., \& Tumlinson, J. 2009, ASSP, 10, 419

Prochaska, J. X., \& Wolfe, A. M. 1996, ApJ, 470, 403

Prochaska, J. X., \& Wolfe, A. M. 1997, ApJ, 487, 73

Prochaska, J. X., \& Wolfe, A. M. 1998, ApJ, 507, 113

Raghunathan, S., Clowes, R. G., Campusano, L. E., et al. 2016, arXiv:1608. 05112

Rahmati, A., \& Schaye, J. 2014, MNRAS, 438, 529

Rao, S. M., Belfort-Mihalyi, M., Turnshek, D. A., et al. 2011, MNRAS, 416, 1215

Rao, S. M., Turnshek, D. A., \& Nestor, D. B. 2006, ApJ, 636, 610

Rauch, M. 1998, A\&A, 36, 267

Rauch, M., Haehnelt, M., Bunker, A., et al. 2008, ApJ, 681, 856

Razoumov, A. O., Norman, M. L., Prochaska, J. X., et al. 2008, ApJ, 683, 149

Ribaudo, J., Lehner, N., Howk, J. C., et al. 2011, ApJ, 743, 207

Rodrigues, L. F. S., Shukurov, A., Fletcher, A., \& Baugh, C. M. 2015, MNRAS, 450, 3472

Rubin, K. H. R., Hennawi, J. F., Prochaska, J. X., et al. 2015, ApJ, 808, 38

Simmons, J. F. L., \& Stewart, B. G. 1985, A\&A, 142, 100

Sokoloff, D. D., Bykov, A. A., Shukurov, A., et al. 1998, MNRAS, 299, 189

Songaila, A., \& Cowie, L. L. 2010, ApJ, 721, 1448

Steidel, C. C. 1993, ASSL, 188, 263

Steidel, C. C., Dickinson, M., Meyer, D. M., Adelberger, K. L., \& Sembach, K. R. 1997, ApJ, 480, 568

Steidel, C. C., \& Sargent, W. L. W. 1992, ApJS, 80, 1

Stil, J. M., Krause, M., Beck, R., \& Taylor, A. R. 2009, ApJ, 693, 1392

Taylor, A. R., Stil, J. M., \& Sunstrum, C. 2009, ApJ, 702, 1230

Tripp, T. M., Meiring, J. D., Prochaska, J. X., et al. 2011, Sci, 334, 952

Tucci, M., \& Toffolatti, L. 2012, AdAst, 2012, 624987

Turnshek, D. A., Monier, E. M., Rao, S. M., et al. 2015, MNRAS, 449, 1536

Vacca, V., Oppermann, N., Enßlin, T., et al. 2016, A\&A, 591, A13

van de Voort, F., Schaye, J., Altay, G., \& Theuns, T. 2012, MNRAS, 421, 2809

Welter, G. L., Perry, J. J., \& Kronberg, P. P. 1984, ApJ, 279, 19

Wise, J. H., \& Abel, T. 2007, ApJ, 665, 899

Wolfe, A. M., \& Chen, H.-W. 2006, ApJ, 652, 981

Wolfe, A. M., Gawiser, E., \& Prochaska, J. X. 2005, A\&A, 43, 861

Wolfe, A. M., Lanzetta, K. M., \& Oren, A. L. 1992, ApJ, 388, 17

Wolfe, A. M., \& Prochaska, J. X. 2000, ApJ, 545, 603

Wolfe, A. M., Prochaska, J. X., \& Gawiser, E. 2003, ApJ, 593, 215 